\title{
HAUSDORFF DIMENSION OF BOUNDARIES OF RELATIVELY HYPERBOLIC GROUPS
}

\author{
LEONID POTYAGAILO AND WEN-YUAN YANG
}

\begin{abstract}
In this paper, we study the Hausdorff dimension of the Floyd and Bowditch boundaries of a relatively hyperbolic group, and show that for the Floyd metric and shortcut metrics respectively, they are are both equal to a constant times the growth rate of the group.

In the proof, we study a special class of conical points called uniformly conical points and establish that, in both boundaries, there exists a sequence of Alhfors regular sets with dimension tending to the Hausdorff dimension and these sets consist of uniformly conical points.
\end{abstract}

\section{INTRODUCTION}

1.1. Main results. The main goal of the paper is to calculate the Hausdorff dimension of the limit set of a geometrically finite action of a finitely generated group $G$ on a compactum $X$. Every action $G \curvearrowright X$ we consider is a convergence action, i.e. the induced action on the space of the distinct triples is discontinuous. We say that $G \curvearrowright X$ is minimal if $X$ coincides with the limit set $\Lambda_{X} G$ (or $\Lambda G$ if $X$ is fixed) of the action, which is the set of the accumulation points of every orbit $G x(x \in X)$.

A point $\xi \in X$ is called conical if there exists a sequence of elements $g_{n} \in G$ $(n \geq 1)$ such that the closure of $\left\{\left(g_{n} \xi, g_{n} \eta\right): n \geq 1\right\}$ in $X^{2}$ is disjoint from the diagonal $\Delta\left(X^{2}\right)=\{(x, x): x \in X\}$ for any $\eta \in X \backslash \xi$. If, in addition, the set of elements $\left\{g_{n} g_{n+1}^{-1}: n \geq 1\right\}$ is in a uniformly bounded distance from the identity, then $\xi$ is called uniformly conical. A quantitative version of an $L$-uniformly conical point for $L \geq 0$ is given in Definition 2.2 .

The action of a subgroup $H<G$ on $X$ is parabolic if $H$ fixes a point $p \in X$, called parabolic fixed point. The parabolic action is bounded parabolic if $H$ acts properly and cocompactly on $X \backslash\{p\}$. We will always assume that the action of the whole group $G$ is non-parabolic so there is no a global fixed point.

A minimal non-parabolic action $G \curvearrowright X$ is called geometrically finite (or relatively hyperbolic) if every point $x \in X$ is either conical or bounded parabolic (cf. Definition 2.2. . The stabilizer of a parabolic point is a maximal parabolic subgroup of $G$. We denote by $\mathcal{P}$ the set of maximal parabolic subgroups and call it peripheral system for the action. A group is called relatively hyperbolic with respect to $\mathcal{P}$ if $G$ admits a geometrically finite action on $X$ with the peripheral system $\mathcal{P}$. If the compactum $X$ on which $G$ acts is metrizable then the action is geometrically finite if and only if the induced action on the space of distinct pairs is co-compact (we say in this case that the action on $X$ is 2-cocompact) [11. If the opposite is not stated

Date: April 11, 2016.

2000 Mathematics Subject Classification. Primary 20F65, 20 F67.

This research is supported by the ERC starting grant GA 257110 RaWG and by ANR grant BLAN2011BS0101304. 
we will always assume that a relatively hyperbolic group is finitely generated and so $X$ is metrizable.

Let $G$ be a group with a finite generating set $S$. Assume that $1 \notin S$ and $S=S^{-1}$. Consider the word metric $d_{S}$ on $G$. Denote $B(n)=\left\{g \in G: d_{S}(1, g) \leq n\right\}$ for $n \geq 0$. The growth rate $\delta_{G, S}$ of $G$ relative to $S$ is the limit

$$
\delta_{G, S}=\lim _{n \rightarrow \infty} \frac{\log \sharp B(n)}{n} .
$$

Recall that Floyd completion of a group $G$ generated by $S$ is the Cauchy completion of the Cayley graph $\mathscr{G}(G, S)$ equipped with the distance $\rho_{\lambda}^{o}$ obtained by rescaling the length of an edge $e \in \mathscr{G}(G, S)$ by a scalar function $\lambda^{d(e, o)}$ for a fixed $\lambda \in] 0,1\left[\right.$ and a basepoint $o \in G$. The distance $\rho_{\lambda}^{o}$ is called Floyd distance at $o$, and we use the notation $\rho$ if $o$ and $\lambda$ are clear from the context (see Subsection 2.2 for more details). We denote by $\bar{G}_{\lambda}$ and $\partial_{\lambda} G$ the corresponding Floyd completion and its boundary respectively. By V. Gerasimov's theorem [12, Proposition 3.4.6] for every finitely generated relatively hyperbolic group the space $\partial_{\lambda} G$ is the universal pullback space for every geometrically finite action of $G \curvearrowright X$ in the sense that there exists an equivariant continuous mapping $F: \partial_{\lambda} G \rightarrow X$ (called Floyd map).

A. Karlsson proved that the action of $G$ on the compact space $\bar{G}_{\lambda}$ is a convergence action [19]. Let $\partial_{\lambda}^{c} G$ (resp. $\partial_{\lambda}^{u c} G$ ) denote the set of all (resp. uniformly) conical points for the action. We denote by $\operatorname{Hdim}_{\rho}$ the Hausdorff dimension with respect to $\rho=\rho_{\lambda, o}$. The first main result of the paper is the following.

Theorem 1.1. Let $G$ be a relatively hyperbolic group with a finite generating set $S$. There exists a constant $0<\lambda_{0}<1$ such that

$$
\operatorname{Hdim}_{\rho}\left(\partial_{\lambda} G\right)=\operatorname{Hdim}_{\rho}\left(\partial_{\lambda}^{c} G\right)=\operatorname{Hdim}_{\rho}\left(\partial_{\lambda}^{u c} G\right)=-\frac{\delta_{G, S}}{\log \lambda}
$$

for any $\lambda \in\left[\lambda_{0}, 1\right)$.

Remark. Note that for a hyperbolic group the Floyd metric is bilipschitz equivalent to the visual metric on the Gromov boundary (with appropriate choices of parameters). Even though this result seems to be a folklore, we have not found the corresponding reference in the literature. We provide a proof of it in the Appendix. As a consequence the result of M. Coornaert [6] for the hyperbolic groups is a partial case of Theorem 1.1 .

Note that the action of $G$ on the Floyd boundary $\partial_{\lambda} G$ is not necessarily geometrically finite, as it is shown in 29] for Dunwoody's inaccessible groups. In particular the Floyd boundary is not in general homeomorphic to the limit set $\Lambda G$. So it is natural to ask if an analogous result to Theorem 1.1 is true for $\Lambda G$.

Consider a minimal geometrically finite action of $G$ on a compact $X=\Lambda G$. It is shown in [13] that the Floyd metric $\rho$ transferred by the Floyd map $F: \partial_{\lambda} G \rightarrow \Lambda G$ is a metric on $\Lambda G$, called shortcut metric, and is denoted by $\bar{\rho}$ (see subsection 2.2 .

Our next goal is to calculate the Hausdorff dimension $\operatorname{Hdim}_{\bar{\rho}}$ of $\Lambda G$ with respect to $\bar{\rho}$. Denote by $\Lambda^{u c} G$ the set of uniformly conical points of $\Lambda G$. The following theorem provides the same conclusion for the shortcut metric as in the case of the Floyd metric.

Theorem 1.2. Let $G$ be a group with a finite generating set $S$ acting geometrically finitely on a compactum $X=\Lambda G$. Then there exists a constant $0<\lambda_{0}<1$ such 
that

$$
\operatorname{Him}_{\bar{\rho}}(\Lambda G)=\operatorname{Hdim}_{\bar{\rho}}\left(\Lambda^{u c} G\right)=-\frac{\delta_{G, S}}{\log \lambda}
$$

for any $\lambda \in\left[\lambda_{0}, 1\right)$.

The above theorems implies the following.

Corollary 1.3. For any shortcut metric $\bar{\rho}$, the Hausdorff dimension of the limit set of every relatively hyperbolic action of a group $G$ is constant and is equal to

$$
\operatorname{Hdim}_{\rho}\left(\partial_{\lambda} G\right)=\operatorname{Hdim}_{\bar{\rho}}(\Lambda G)=-\frac{\delta_{G, S}}{\log \lambda}
$$

for any $\lambda \in\left[\lambda_{0}, 1\left[\right.\right.$ where $\left.\lambda_{0} \in\right] 0,1[$ is a fixed number.

We say that a metric space $X$ is Ahlfors $Q$-regular for a constant $Q>0$ if there exists a Borel measure $\mu$ on $X$ such that the following holds

$$
\mu(B(x, r)) \asymp r^{Q}
$$

for any open ball $B(x, r)$ centered at $x \in X$ of radius $r>0$, where the symbol $\asymp$ denotes the bilipschitz equivalence between two quantities: $C^{-1} r^{Q} \leq \mu(B(x, r)) \leq$ $C r^{Q}$ for a uniform constant $C$.

Our next main result shows that the Hausdorff dimension of the Floyd boundary and of the limit set of a relatively hyperbolic action can be well-approximated by a sequence of Ahlfors regular subsets.

Theorem 1.4. Let $G$ be a finitely generated relatively hyperbolic group with a finite generating set $S$. Then there exists a sequence of Ahlfors $Q_{i}$-regular subsets $X_{i}$ in $\partial_{\lambda} G$ or $\Lambda G$ such that $X_{i}$ consists of uniformly conical points, $0<Q_{i}<\delta_{G, S}$ and $Q_{i} \rightarrow \delta_{G, S}$ as $i \rightarrow \infty$.

The proof of Theorem 1.4 is based on the existence of an $L$-transitional geodesic tree $\mathcal{T}=\mathcal{T}(L) \subset G$ (Lemma 3.7) depending on a parameter $L \gg 0$. Every vertex of $\mathcal{T}$ is a central point of a geodesic interval, whose size depends on $L$, and which belongs to a neighbourhood of a left coset (horosphere) $g P$ where $P \in \mathcal{P}$ (see Subsection 2.4). We show that the endpoints of such a tree are $L$-uniformly conical (Lemma 2.12). However it is not true in general that every uniformly conical point appears as an endpoint of an $L$-transitional tree for a bounded $L$ (see the discussion after Lemma 2.12). The proof of Theorem 1.4 shows that the Hausdorff dimension of the endpoints of $L$-transitional trees well approximate the Hausdorff dimension of the Floyd boundary (or the limit set) if $L \rightarrow \infty$. We recapitulate all these facts in the following.

Corollary 1.5. There exists a sequence $T_{i}$ of $L_{i}$-transitional trees such that $X_{i}=$ $\partial T_{i}$ are Ahlfors $Q_{i}$-regular spaces from the statement of Theorem 1.4.

Our next result given in Section 5 generalizes the result of 14 that the geodesics of the Floyd metrics are approximated by so called tight paths. Considering generalized tight paths (see Definition 5.9) we show that they approximate the geodesics with respect to the shortcut metric defined on the limit set.

Let $\partial_{L, o}^{u c} G$ and $\Lambda_{L, o}^{u c} G$ denote subsets of uniformly conical points in $\partial_{\lambda}^{u c} G$ and $\Lambda^{u c} G$ depending on the above parameter $L$ (see Subsection 2.4 for the precise definitions). The following result is central in this Section. 
Theorem 1.6 (Proposition 5.13). Under the assumptions of Theorem 1.1 there exists $0<\lambda_{0}<1$ such that for any $L>0$ and $\lambda \in\left[\lambda_{0}, 1[\right.$ we have

$$
\rho_{\lambda, o}(\xi, \eta) \asymp_{L} \lambda^{n}, \forall \xi \neq \eta \in \partial_{L, o}^{u c} G
$$

and

$$
\bar{\rho}_{\lambda, o}(\xi, \eta) \asymp_{L} \lambda^{n}, \forall \xi \neq \eta \in \Lambda_{L, o}^{u c} G,
$$

where $n=d(o,[\xi, \eta])$.

1.2. Historical remarks and motivations. We provide here a short history of the study of the Hausdorff dimension of the limit set of various convergence actions: Kleinian, hyperbolic and relatively hyperbolic.

The identification of the Hausdorff dimension with the critical exponent of Poincaré series was first established by S. Patterson 21. He introduced a probability measure on the limit set of the convex-cocompact Fuchsian groups, and proved that up to a constant it is equal to the Hausdorff measure. D. Sullivan generalized this result and constructed such measures (called since then Patterson-Sullivan measures) on the limit sets of geometrically finite Kleinian groups acting on the hyperbolic space $\mathbb{H}^{n}$ of dimension $n$ 25]. To finish the discussion of the case of Kleinian groups, we note the result of C. Bishop and P. Jones who proved in [1] that for a non-elementary Kleinian group acting on the hyperbolic 3-space the Hausdorff dimension of the conical limit set is equal to the critical exponent of the Poincaré series (compare with our Theorems 1.1 and 1.2). The latter results were generalized by F. Paulin 22 to discrete groups of isometries of Riemannian manifolds of strictly negative curvature.

M. Coornaert has generalized the results of Patterson-Sullivan to the class of word-hyperbolic groups [6]. In particular he proved that the Hausdorff dimension of the (Gromov) boundary of such a group with respect to the visual metric is equal to the critical exponent of the Poincare series.

A natural question arises whether Coornaert's theorem holds for the class of relatively hyperbolic groups. However it was shown by M. Burger and S. Mozes that if $G$ is a closed subgroup of the isometry group of a CAT $(-1)$ space $X$ and the parabolic subgroups of $G$ are not amenable then the critical exponent is infinite [4, Proposition 1.6]. Such an example of a relatively hyperbolic group whose parabolic subgroups contain non-cyclic free subgroups was constructed by D. Gaboriau and F. Paulin [10, Example 1, p. 189]. By [22] it then follows that the Hausdorff dimension of the limit set for the action of such a group with respect to the visual metric is infinite too. So in order to generalize Coornaert's theorem to the class of relatively hyperbolic groups one must replace the visual metric by a different one.

The Floyd metric obtained by a rescaling procedure of the word metric is a natural candidat as it extends to the Floyd compactification of a group. Furthermore by a theorem of V. Gerasimov there exists an equivariant and continuous map from the Floyd boundary $\partial_{\lambda} G$ to the limit set of any relatively hyperbolic action of $G[12$. In particular, if $G$ is hyperbolic, the Floyd and Gromov boundaries are bilipschitz equivalent for some exponential Floyd function.

M. Bourdon has observed (private communication) that the Hausdorff dimension of the Floyd boundary of a relatively hyperbolic group, calculated with respect to the Floyd metric obtained with the exponential rescaling function $\lambda^{n}(\lambda \in(0,1))$ is always upper bounded by $-\frac{\delta_{G, S}}{\log \lambda}$ (cf. Lemma 4.1). However the question whether it 
admits a lower strictly positive bound, which is equal to the same constant remained open. This was our first motivation giving rise to Theorem 1.1 . Theorem 1.2 is then obtained by transferring the Floyd metric from the Floyd boundary $\partial_{\lambda} G$ to the limit set $\Lambda G$ of the geometrically finite action using the above Gerasimov's map.

The lower bound estimate for the Hausdorff dimension in Theorems 1.1 and 1.2 follow from Theorem 1.4 providing the approximation of the boundary points by Ahlfors regular subsets $X_{i}$. These subsets entirely consist of uniformly conical points which are the space of ends of subtrees of the Cayley graph of $G$. Note that the idea of such an approximation by trees is quite standard in both settings: hyperbolic (see e.g. [18, 6.1]) or Kleinian (see [1). However these constructions of trees essentially use the hyperbolicity of the ambiant space. The latter property is not true for a relatively hyperbolic group: the Cayley graph is not in general hyperbolic and the relative Cayley graph is hyperbolic but the action on the set of vertices is not proper. The approximating trees constructed in the paper admits certain periodicity allowing us to obtain a Patterson-Sullivan measure $\mu$ on $X_{i}$ also having periodic properties. Theorem 1.4 then shows that these measures converge to the Hausdorff measure on a subset of uniformly conical points and whose dimension coincides with the full Hausdorff dimension of the ambiant space.

Acknowledgments. The authors are deeply grateful to Marc Bourdon for several discussions motivating our initial interest to the subject of the paper, we owe the proof of Lemma 4.1 to him as well as the references to the papers [4] and 10.

We also thank Misha Kapovich who indicated us that the bilipschitz equivalence between the Floyd and the visual metrics needs to be justified (see the Remark above).

During the work on this paper the first author was partially supported by the ANR grant DiscGroup (BLAN 2011 BS01 013 04). He is also very grateful to the Max-Planck-Institut für Mathematik in Bonn for the hospitality and support during his research stay at the Institute where a part of the work was done.

The second author is grateful to the CNRS for providing him a research fellowship at the University of Lille 1, and was partially supported by the ERC starting grant GA 257110 RaWG when he was a postdoc in Orsay.

\section{Preliminaries}

2.1. Notations and Conventions. Let $(Y, d)$ be a geodesic metric space. Given a subset $X$ and a number $r \geq 0$, let $N_{r}(X)=\{y \in Y: d(y, X) \leq r\}$. For $x \in Y$ denote $B(x, r)=N_{r}(\{x\})$. Sometimes, we will write $B_{d}(x, r)$ to emphasize the metric $d$.

Given a point $y \in Y$ and a subset $X \subset Y$, let $\operatorname{Proj}_{X}(y)$ be the set of points $x$ in $X$ such that $d(y, x)=d(y, X)$. The projection of a subset $A \subset Y$ to $X$ is then $\operatorname{Proj}_{X}(A)=\cup_{a \in A} \operatorname{Proj}_{X}(a)$.

We always consider a rectifiable path $\alpha$ in $Y$ with arc-length parametrization. Denote by $\ell(\alpha)$ the length of $\alpha$, and by $\alpha_{-}, \alpha_{+}$the initial and terminal points of $\alpha$ respectively. Let $x, y \in \alpha$ be two points which are given by parametrization. Then denote by $[x, y]_{\alpha}$ the parametrized subpath of $\alpha$ going from $x$ to $y$. We also denote by $[x, y]$ a choice of a geodesic in $Y$ between $x, y \in Y$.

A path $\alpha$ is called a $c$-quasi-geodesic for $c \geq 1$ if the following holds

$$
\ell(\beta) \leq c \cdot d\left(\beta_{-}, \beta_{+}\right)+c
$$


for any rectifiable subpath $\beta$ of $\alpha$.

Let $\alpha, \beta$ be two paths in $Y$. Denote by $\alpha \cdot \beta$ (or simply $\alpha \beta$ ) the concatenated path provided that $\alpha_{+}=\beta_{-}$.

A path $\alpha$ going from $\alpha_{-}$to $\alpha_{+}$induces a first-last order as follows. Given a property $(\mathrm{P})$, a point $z$ on $\alpha$ is called the first point satisfying $(\mathrm{P})$ if $z$ is among the points $w$ on $\alpha$ with the property $(\mathrm{P})$ such that $\ell\left(\left[\alpha_{-}, w\right]_{\alpha}\right)$ is minimal. The last point satisfying $(\mathrm{P})$ is defined in a similar way (replacing $\left[\alpha_{-}, w\right]_{\alpha}$ by $\left[w, \alpha_{+}\right]_{\alpha}$ ).

Let $f, g$ be real-valued functions with domain understood in the context. Then $f \prec_{c_{i}} g$ means that there is a constant $C>0$ depending on parameters $c_{i}$ such that $f<C g$, and $\succ_{c_{i}}$ is defined similarly. We use the symbol $\asymp_{c_{i}}$ if both inequalities are true. For simplicity, we omit $c_{i}$ if they are some universal constants.

Denote by $\|\cdot\|$ the diameter of a set in a metric space. Recall the notion of Hausdorff measures in a metric space.

Definition 2.1. Let $X$ be a subset in a metric space $(Y, d)$. Given numbers $\epsilon, s \geq 0$, define

$$
\mathcal{H}_{\epsilon}^{s}(X)=\inf \left\{\sum\left\|U_{i}\right\|^{s}: X \subset \bigcup_{i=1}^{\infty} U_{i}, U_{i} \subset Y,\left\|U_{i}\right\| \leq \epsilon\right\} .
$$

Define $\mathcal{H}^{s}(X)=\lim _{\epsilon \rightarrow 0} \mathcal{H}_{\epsilon}^{s}(X)$, the $s$-dimensional Hausdorff measure of $X$. The Hausdorff dimension of $X$ is defined as follows,

$$
\operatorname{Hdim}_{d}(X)=\inf \left\{s \geq 0: \mathcal{H}^{s}(X)=0\right\}=\sup \left\{s \geq 0: \mathcal{H}^{s}(X)=\infty\right\} .
$$

By convention, set $\inf \emptyset=\sup \left\{s \in \mathbb{R}_{\geq 0}\right\}=\infty$. Thus, $\operatorname{Hdim}_{d} X \in[0, \infty]$. Note that $\mathcal{H}^{s}(X)$ may be zero for $s=\operatorname{Hdim}_{d} X$.

2.2. Floyd boundary and relative hyperbolicity. Let $G$ be a group with a finite generating set $S$. Assume that $1 \notin S$ and $S=S^{-1}$. Let $\mathscr{G}(G, S)$ be the Cayley graph of $G$ with respect to $S$. Denote by $d_{S}$ (or simply by $d$ if there is no ambiguity) the word metric on $\mathscr{G}(G, S)$.

Fix $0<\lambda<1$ and a basepoint $o \in G$. We define a Floyd metric $\rho_{\lambda, o}$ as follows. The Floyd length $\mathfrak{l}_{\lambda, o}(e)$ of an edge $e$ in $\mathscr{G}(G, S)$ is $\lambda^{n}$, where $n=d(o, e)$. The Floyd length $\mathfrak{l}_{\lambda, o}(\gamma)$ of a path $\gamma$ is the sum of Floyd lengths of its edges. This induces a length metric $\rho_{\lambda, o}$ on $\mathscr{G}(G, S)$, which is the infimum of Floyd lengths of all possible paths between two points.

Let $\bar{G}_{\lambda}$ be the Cauchy completion of $G$ with respect to $\rho_{\lambda, o}$. The complement $\partial_{\lambda} G$ of $\mathscr{G}(G, S)$ in $\bar{G}_{\lambda}$ is called Floyd boundary of $G$. The $\partial_{\lambda} G$ is called non-trivial if $\sharp \partial_{\lambda} G>2$. We refer the reader to [9], 12], [13, [19] for more details.

By construction, the following equivariant property holds

$$
\rho_{\lambda, o}(x, y)=\rho_{\lambda, g o}(g x, g y)
$$

for any $g \in G$. The Floyd metrics with different basepoints are related by a biLipschitz inequality:

$$
\lambda^{d\left(o, o^{\prime}\right)} \leq \frac{\rho_{\lambda, o}(x, y)}{\rho_{\lambda, o^{\prime}}(x, y)} \leq \lambda^{-d\left(o, o^{\prime}\right)}
$$

for any two points $o, o^{\prime} \in G$.

We now recapitulate few standard definitions concerning geometrically finite convergence actions which will be often used further. 
Definition 2.2. Let $X$ be a compact metrizable space on which $G$ admits a minimal and non-trivial convergence action by homeomorphisms.

(1) A point $\xi \in X$ is called conical if there exists a sequence of elements $g_{n} \in G$ $(n \geq 1)$ such that the closure of $\left\{g_{n}(\xi, \eta): n \geq 1\right\}$ in $X^{2}$ is disjoint from the diagonal $\Delta\left(X^{2}\right)=\{(x, x): x \in X\}$ for any $\eta \in X \backslash \xi$.

If, in addition, there exists $L>0$ such that $d\left(1, g_{n} g_{n+1}^{-1}\right) \leq L$, then $\xi$ is called $L$-uniformly conical (or uniformly conical if the constant $L$ is not important).

(2) A point $\xi \in X$ is called bounded parabolic if the stabilizer $G_{\xi}$ of $\xi$ in $G$ is infinite, and acts properly and co-compactly on $X \backslash \xi$. The subgroup $G_{\xi}$ is called maximal parabolic.

(3) A convergence group action of $G$ on $X$ is called geometrically finite if every limit point $\xi \in X$ is either a conical point or a bounded parabolic point.

As it was mentioned in the Introduction a pair $(G, \mathcal{P})$ is relatively hyperbolic if $G$ admits a geometrically finite group action on a compact metrizable space $X$ such that $\mathcal{P}$ coincides with the collection of maximal parabolic subgroups (peripheral system). Using the relative Cayley graph one can construct the limit set $\Lambda G$ of the action with the boundary of this graph 3 . We will often call Bowditch boundary the limit set $\Lambda G$ of a geometrically finite action. Bowditch proved that if $G$ is finitely generated then $\Lambda G$ up to an equivariant homeomorphism depends only on the pair $(G, \mathcal{P})[2$. We also note the same result still holds in general case when $G$ is not finitely generated [16, Corollary 6.1.e].

The following result establishes the following universal pullback property of the Floyd boundary.

Proposition 2.3. 12 Suppose $(G, \mathcal{P})$ is a relatively hyperbolic pair. Then there exists $0<\lambda_{0}<1$ such that for any $\lambda \in\left[\lambda_{0}, 1\right)$ there exists a continuous $G$-equivariant surjective map (called Floyd map):

$$
F_{\lambda}: \partial_{\lambda} G \rightarrow \Lambda G
$$

Let $G_{p}$ be the stabilizer of a parabolic point $p \in X$ for the action $G \curvearrowright X=\Lambda G$. Denote by $\Lambda_{\partial_{\lambda} G}\left(G_{p}\right)$ and $\partial_{\lambda} G_{p}$ the limit set of $G_{p}$ for its action on the Floyd boundary $\partial_{\lambda} G$ of $G$, and the Floyd boundary of $G_{p}$ respectively. The following result precisely describes the kernel of the Floyd map.

Proposition 2.4. 13. Under the assumption of Proposition 2.3, the following holds

$$
F_{\lambda}^{-1}(p)=\Lambda_{\partial_{\lambda} G}\left(G_{p}\right)=\partial_{\lambda} G_{p}
$$

for any parabolic point $p$ in $\Lambda$. Moreover, $F_{\lambda}^{-1}(p)$ consists of one point if $p$ is a conical point.

We equip $\Lambda G$ with a shortcut metric as follows: let

$$
\omega=\left\{(\eta, \xi) \in \partial_{\lambda} G \times \partial_{\lambda} G: F_{\lambda}(\xi)=F_{\lambda}(\eta)\right\}
$$

be the relation on $\partial_{\lambda} G$ given by the Floyd map $F_{\lambda}: \partial_{\lambda} G \rightarrow \Lambda G$. For any $\xi, \eta \in \bar{G}_{\lambda}$, define a pseudo-distance $\tilde{\rho}_{\lambda, o}(\xi, \eta)$ on $\bar{G}_{\lambda}$ to be

$$
\tilde{\rho}_{\lambda, o}(\xi, \eta)=\inf _{n \geq 1}\left\{\sum_{i=1}^{n} \rho_{\lambda, o}\left(\xi_{i}, \eta_{i}\right):\left(\eta_{i}, \xi_{i+1}\right) \in \omega, 1 \leq i<n, \xi_{1}=\xi, \eta_{n}=\eta\right\} .
$$


We have

$$
\forall \xi, \eta \in \bar{G}_{\lambda}: \tilde{\rho}_{\lambda, o}(\xi, \eta) \leq \rho_{\lambda, o}(\xi, \eta),
$$

and it is a maximal pseudo-metric on $\bar{G}_{\lambda} \times \bar{G}_{\lambda}$ satisfying this inequality. It is shown in 12 that the space $\widetilde{\Lambda G}:=\Lambda G \sqcup \mathscr{G}(G, S)$ (called attractor sum) is compact. The action $G \curvearrowright \widetilde{\Lambda G}$ is convergence such that its restriction on $\mathscr{G}(G, S)$ is the identity and on $\Lambda G$ it coincides with the initial action. Furthermore the Floyd map $F_{\lambda}$ extends to an equivariant continuous map (denoted by the same symbol):

$$
F_{\lambda}: \bar{G}_{\lambda} \rightarrow \widetilde{\Lambda G}
$$

such that $\left.F_{\lambda}\right|_{G} \equiv$ id. Pushing forward $\tilde{\rho}_{\lambda, o}$ with $F_{\lambda}$, we obtain a shortcut pseudometric on $\widehat{\Lambda G}$ :

$$
\forall x, y \in \widetilde{\Lambda G}: \bar{\rho}_{\lambda, o}(x, y)=\tilde{\rho}_{\lambda, o}\left(F_{\lambda}^{-1}(x), F_{\lambda}^{-1}(y)\right),
$$

which turns out to be a real metric on $\widetilde{\Lambda G}$ (see [13, Section 3] for details). By the above construction, one can easily see that the shortcut metrics $\bar{\rho}_{\lambda, o}$ satisfy the properties (1) and (2) too.

Convention 2.5. Since now on we will always suppose that $\lambda \in\left[\lambda_{0}, 1\right)$ where $\lambda_{0}$ is given by Proposition 2.3. We omit the index $\lambda$ in $\mathfrak{l}_{\lambda, o}, \rho_{\lambda, o}$ and $\bar{\rho}_{\lambda, o}$ if $\lambda$ is given in the context.

Finally, we recall the following Visibility Lemma.

Lemma 2.6 (Visibility lemma). 19 There is a function $\varphi: \mathbb{R}_{\geq 0} \rightarrow \mathbb{R}_{\geq 0}$ such that for any $v \in G$ and any geodesic $\gamma$ in $\mathscr{G}(G, S)$, we have if $\mathfrak{l}_{v}(\gamma) \geq \kappa$, then $d(v, \gamma) \leq \varphi(\kappa)$.

Remark. The same result is valid for quasi-geodesics or more general $\Theta$-geodesics where $\Theta: \mathbb{N} \rightarrow G$ is a polynomial distortion function [13, Lemma 5.1].

2.3. Floyd geodesics. In this subsection, we provide a few basic tools to study Floyd geodesics.

We say that a path $\alpha: \mathbb{Z} \rightarrow \mathscr{G}(G, S)$ ends at $\xi \in \partial_{\lambda} G$ if $\xi=\lim _{n \rightarrow \infty} \alpha(n)$. Denote in this case $\alpha_{+}=\xi$, and $\alpha_{-}=\lim _{n \rightarrow-\infty} \alpha(n)$. It follows from Lemma 2.6 that every geodesic ray ends at a point of the Floyd boundary. Moreover, $\bar{G}_{\lambda}$ is a geodesic metric space and is a visual boundary: any two distinct points $\xi, \eta \in \bar{G}_{\lambda}$ are connected by a bi-infinite word geodesic belonging to the Cayley graph [13, Proposition 2.4].

We note that a Floyd geodesic between $\xi, \eta$ does not necessarily belong to the graph (e.g. an example of such situation is given by the Floyd geodesic $[n,+\infty] \cup$ $[-\infty,-n]$ between $-n$ and $n$ for the group $\mathbb{Z}+\mathbb{Z})$. A method to overcome this problem was proposed in 14. It consists in introducing a special type of paths called tight paths (see Definition 5.1 in section 5p situating in the Cayley graph which will approximate well the Floyd geodesics. To provide a certain development of this method we will need the following preliminary statements.

Lemma 2.7. [14, Lemma 7.2] For any $l>0$, there exists $0<\lambda_{0}<1$ such that the following property hold for any $\lambda \in\left[\lambda_{0}, 1\right)$.

Let $x, y \in \mathscr{G}(G, S)$ such that $d(x, y) \leq l$, and $p$ be a path with $\alpha_{-}=x$ such that $\ell(\alpha) \geq d(x, y)+1$. Then $\mathfrak{l}_{\lambda, o}(\alpha)>\rho_{\lambda, o}(x, y)$. In particular, the $\rho_{\lambda, o}$-geodesic between $x, y$ is a geodesic in $\mathscr{G}(G, S)$ 
We consider the following shortening procedure introduced in [14]: consider two points $x, y \in \bar{G}_{\lambda}$, we take a sequence of paths $\gamma_{n}$ in $\mathscr{G}(G, S)$ such that $\left(\gamma_{n}\right)_{-} \rightarrow$ $x,\left(\gamma_{n}\right)_{+} \rightarrow y$ and

$$
\mathfrak{l}_{\lambda, o}\left(\gamma_{n}\right) \rightarrow \rho_{\lambda, o}(x, y)
$$

For every $l>0$ we can choose $\left.\lambda_{0} \in\right] 0,1\left[\right.$ such that $\gamma_{n}$ is an $l$-local geodesic. Indeed, if a segment between two points of $\gamma_{n}$ at distance at most $l$ is not a geodesic, then it can be replaced by a geodesic. Applying this procedure several times, we obtain a $l$-local geodesic, still denoted by $\gamma_{n}$, whose Floyd length is not increased by Lemma 2.7 (see Lemma 5.8 for more details).

The following lemma states that word geodesic rays are also Floyd and shortcut geodesics.

Lemma 2.8. Let $o \in G$ be a base point and $\gamma$ be a geodesic ray with $\gamma_{-}=o$. Then for any $v \in \gamma$ we have

$$
\mathfrak{l}_{\lambda, o}\left([v, x]_{\gamma}\right)=\bar{\rho}_{\lambda, o}(v, y)
$$

and

$$
\mathfrak{l}_{\lambda, o}\left([v, x]_{\gamma}\right)=\rho_{\lambda, o}(v, x)
$$

where $x=\gamma_{+} \in \partial_{\lambda} G$ and $y=F(x) \in \Lambda G$ where $F$ is the Floyd map given in Proposition 2.3 .

Proof. We only prove the result for the shortcut metric. In the case of Floyd metric a straightforward calculation shows that the geodesic ray $\gamma$ as well as every its subray is also a $\rho_{\lambda, o}$-Floyd geodesic.

By definition (3) of a shortcut metric, for any $n \in \mathbb{N}$, there exist pairs $\left(\eta_{i}, \xi_{i+1}\right) \in$ $\omega$ where $1 \leq i<m$ such that

$$
\bar{\rho}_{\lambda, o}(v, y) \geq \sum_{1 \leq i \leq m} \rho_{\lambda, o}\left(\xi_{i}, \eta_{i}\right)-\frac{1}{2 n},
$$

where $\xi_{1}=v, \eta_{m}=x$. Every geodesic ray $\left[o, \eta_{1}\right]$ is also a Floyd geodesic so we can choose $\tilde{\eta}_{1} \in\left[o, \eta_{1}\right]$ such that $\rho_{\lambda, o}\left(\tilde{\eta}_{1}, \eta_{1}\right) \leq \frac{1}{2 n}$. It follows

$$
\bar{\rho}_{\lambda, o}(v, y) \geq \rho_{\lambda, o}\left(v, \tilde{\eta}_{1}\right)-\frac{1}{n} .
$$

Choose $w \in[v, y]_{\gamma}$ such that $d(v, w)=d\left(v, \tilde{\eta}_{1}\right)=m$. Then the following is true:

$$
\rho_{\lambda, o}\left(v, \tilde{\eta}_{1}\right) \geq \rho_{\lambda, o}(v, w) .
$$

Indeed, connect $v$ and $\tilde{\eta}_{1}$ by a curve $\alpha$. There exists a point $u=\alpha\left(t_{0}\right)$ such that $d(v, u)=m$ and choose a sub-curve $\alpha^{\prime}=[v, u]_{\alpha}$ containing $m$ edges. Since $\gamma$ is a word geodesic, for the k-th edge $e \in \alpha^{\prime}$ and the $k$-th edge $e_{1} \in[v, w]_{\gamma}$ we have $\mathfrak{l}_{\lambda, o}(e) \geq \mathfrak{l}_{\lambda, o}\left(e_{1}\right)(k \in\{0, \ldots, m\})$. Then $\mathfrak{l}_{\lambda, o}(\alpha) \geq \mathfrak{l}_{\lambda, o}\left(\alpha^{\prime}\right) \geq \rho_{\lambda, o}(v, w)$. So (6) follows. 


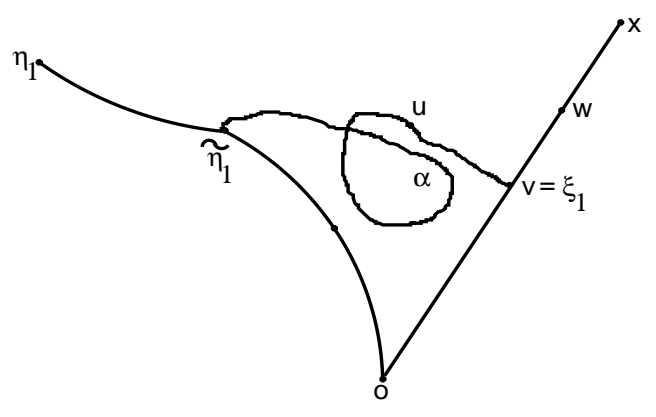

We have

$$
\begin{aligned}
\bar{\rho}_{\lambda, o}(v, y) & \geq \rho_{\lambda, o}\left(v, \tilde{\eta}_{1}\right)-\frac{1}{2 n} \geq \rho_{\lambda, o}(v, w)-\frac{1}{n} \geq \\
& \geq \mathfrak{l}_{\lambda, o}\left([v, x]_{\gamma}\right)-\frac{\lambda^{n}}{1-\lambda}-\frac{1}{n} .
\end{aligned}
$$

Passing to the limit we obtain $\bar{\rho}_{\lambda, o}(v, y) \geq \mathfrak{l}_{\lambda, o}\left([v, x]_{\gamma}\right)=\rho_{\lambda, o}(v, x)$. Since $\rho_{\lambda, o}(v, x) \geq \bar{\rho}_{\lambda, o}(v, y)$, we conclude that $\mathfrak{l}_{\lambda, o}\left(\left[v, \gamma_{+}\right]_{\gamma}\right)=\bar{\rho}_{\lambda, o}\left(v, \gamma_{+}\right)$.

2.4. Transitional paths and uniformly conical points. In this subsection we shall give a description of uniformly conical points in $\Lambda G$ using the geometry of Cayley graph.

Let $(G, \mathcal{P})$ be a relatively hyperbolic pair. Denote $\mathbb{P}=\{g P: g \in G, P \in \tilde{\mathcal{P}}\}$, where $\tilde{\mathcal{P}}$ is a maximal set of non-conjugate subgroups in $\mathcal{P}$. Following [13] we call the elements of $\mathbb{P}$ horospheres.

Definition 2.9. Fix $\epsilon, R>0$. Let $\gamma$ be a path in $\mathscr{G}(G, S)$ and $v \in \gamma$ a vertex. Given $X \in \mathbb{P}$, we say that $v$ is $(\epsilon, R)$-deep in $X$ if

$$
\gamma \cap B(v, R) \subset N_{\epsilon}(X) .
$$

If $v$ is not $(\epsilon, R)$-deep in any $X \in \mathbb{P}$, then $v$ is called an $(\epsilon, R)$-transition point of $\gamma$.

The following lemma together with Lemma 2.6 will be invoked several times.

Lemma 2.10. (1) For any $c \geq 1, R>0$, there exists $\epsilon=\epsilon(c), \kappa=\kappa(\epsilon, R)>0$ such that for any c-quasi-geodesic $\gamma$ and an $(\epsilon, R)$-transitional point $v$ in $\gamma$, we have

$$
\rho_{v}\left(\gamma_{-}, \gamma_{+}\right) \geq \bar{\rho}_{v}\left(\gamma_{-}, \gamma_{+}\right)>\kappa .
$$

(2) For any $c \geq 1, \kappa, \epsilon>0$ there exists $R=R(c, \kappa, \epsilon)>0$ such that for any c-quasi-geodesic $\gamma$ and a point $v \in \gamma$ with $\bar{\rho}_{v}\left(\gamma_{-}, \gamma_{+}\right)>\kappa$, we have that $v$ is an $(\epsilon, R)$-transitional point of $\gamma$.

Proof. Let us first prove (2). Suppose not, then $\exists c \geq 1, \kappa, \epsilon>0: \forall n, \exists c$-quasigeodesics $\gamma_{n}$ and $v_{n} \in \gamma_{n}$ such that $v_{n}$ is $(\epsilon, n)$-deep and $\bar{\rho}_{v_{n}}\left(\left(\gamma_{n}\right)_{-},\left(\gamma_{n}\right)_{+}\right)>\kappa$. Up to a normalization we may assume that $v_{n}=v=\gamma_{n}(0)$. Then $\gamma_{n}(]-n, n[) \subset$ $N_{\epsilon}\left(X_{n}\right)$ for $X_{n} \in \mathbb{P}$. By compactness of geodesics in the Tikhonoff topology, we obtain a limit horocycle $\alpha$ such that $\alpha_{ \pm}=q$ and every part of $\alpha$ belongs to $\gamma_{n}$ for sufficiently large $n$ (see [14. Prop. 5.2.3] for more details). Then the diameter of $\partial\left(\gamma_{n} \cap \alpha\right)$ with respect to the distance $\bar{\rho}_{v}$ tends to 0 . As $\gamma_{n}$ are geodesics whose 
all interior points are in the graph we must have $\bar{\rho}_{v}\left(\left(\gamma_{n}\right)_{-},\left(\gamma_{n}\right)_{+}\right) \rightarrow 0$ which is a contradiction.

(1). By [13, Corollary 3.9] there exists a constant $\epsilon=\epsilon(c)$ such that for every $X \in \mathbb{P}$ any $c$-quasi-geodesic with endpoints in $X$ lies in $N_{\epsilon}(X)$ (all horospheres are uniformly quasi-convex). For the constants $c$ and $\epsilon=\epsilon(c)$ the statement now follows from [15, Corollary 5.10], following a similar argument as above.

We introduce a special class of paths, which plays an important role in the present study.

Definition 2.11. Given $\epsilon, R, L>0$, a path $\gamma$ in $\mathscr{G}(G, S)$ is called $(\epsilon, R, L)$-transitional (or simply transitional if the choice of the constants is not important) if for any point $v \in \gamma$, there exists an $(\epsilon, R)$-transitional point $w \in \gamma$ such that $\ell\left([v, w]_{\gamma}\right) \leq L$.

We say that an infinite path $\gamma$ in $\mathscr{G}(G, S)$ is eventually $(\epsilon, R, L)$-transitional if there exists $v \in \gamma$ such that $\left[v, \gamma_{+}\right)_{\gamma}$ is $(\epsilon, R, L)$-transitional.

We fix the constant $\epsilon=\epsilon(1)>0$ given by Lemma 2.10 1 . The following lemma characterizes uniformly conical points as the endpoints of transitional geodesic rays.

Lemma 2.12. Let $(G, \mathcal{P})$ be a relatively hyperbolic pair. There exists $R>0$ for which tplhe following property is true:

a point $\xi \in \Lambda G$ is uniformly conical if and only if some (or any) geodesic ray ending at $\xi$ is eventually an $(\epsilon, R, L)$-transitional geodesic ray for some $L>0$.

Proof of " $\Rightarrow$ ". Since $G$ acts geometrically finitely on $\Lambda G$, it follows from [26, Theorem $1 \mathrm{C}]$ that there exists $\delta>0$ such that for any conical point $\xi \in \Lambda G$, there exists a sequence $\left(g_{n}\right) \subset G$ such that for all points $\eta \in(\Lambda G \cup G) \backslash \xi$ one has $\bar{\rho}_{1}\left(g_{n} \xi, g_{n} \eta\right)>\delta$. Denote $r_{0}:=\varphi(\delta / 2)$, where $\varphi$ is given by Lemma 2.6 .

Assume that $\xi$ is an $L$-uniformly conical point for some $L>0$. Let $\gamma=\left[\gamma_{-}, \xi[\right.$ be a geodesic ray ending at $\xi$ and $\left(g_{n}\right) \subset G$ be the above sequence taken for the pair $\left(\gamma_{-}, \xi\right)$. Then $\bar{\rho}_{1}\left(g_{n} \xi, g_{n} \gamma_{-}\right)=\bar{\rho}_{g_{n}^{-1}}\left(\xi, \gamma_{-}\right)>\delta / 2$ and $d\left(1, g_{n} g_{n+1}^{-1}\right) \leq L$ for all $n \geq 1$. By Lemma 2.6, $\gamma \cap B\left(g_{n}^{-1}, r_{0}\right) \neq \emptyset$ for $n \geq 1$. Let $v_{n} \in \gamma$ such that $d\left(v_{n}, g_{n}^{-1}\right)<r_{0}$. By the inequality (2) such that $\bar{\rho}_{v_{n}}\left(\gamma_{-}, \xi\right)>\kappa$ where $\kappa=\lambda^{r_{0}} \cdot \delta / 2$ is a uniform constant. Moreover, $d\left(v_{n}, v_{n+1}\right) \leq L+2 r_{0}$.

Hence, Lemma 2.10, 2 gives rise to a uniform constant $R$ for which $v_{n}$ are all $(\epsilon, R)$-transitional for $n \geq 1$.

Proof of " $\Leftarrow$ ". Let $\gamma$ be an $(\epsilon, R, L)$-transitional geodesic ray at $\xi=\gamma_{+}$, and $v_{n}(n \geq 0)$ a sequence of $(\epsilon, R)$-transitional points in $\gamma$ such that $d\left(v_{n}, v_{n+1}\right) \leq L$ and $v_{n} \rightarrow \xi$. Then $\bar{\rho}_{v_{n}}\left(\gamma_{-}, \xi\right) \geq \kappa$, where $\kappa>0$ is given by Lemma 2.101. Denote $g_{n}:=v_{n}^{-1}$. Then $\bar{\rho}_{1}\left(g_{n} \gamma_{-}, g_{n} \xi\right) \geq \kappa$. In other words, $\left\{\left(g_{n} \gamma_{-}, g_{n} \xi\right)\right\}$ lies outside a uniform neighborhood of the diagonal $\Delta\left(\Lambda G^{2}\right)$.

Since the action is convergence the point $\xi$ is conical. As $d\left(1, g_{n} g_{n+1}^{-1}\right) \leq L$ it is uniformly conical.

Remarks. (1) The proof of the " $\Leftarrow$ " direction equally applies to a conical point in Floyd boundary $\partial_{\lambda} G$ without assuming the geometrical finiteness of the action.

(2) The existence of the uniform constant $\delta>0$ which measures the size of a compact fundamental set for the co-compact action of $G$ on the set of distinct pairs was only used to prove the implication " $\Rightarrow$ " (in order to get a uniform constant $R$ ). The existence of such a constant implies that the action of $G$ on a metrizable space $\Lambda G$ is 2-cocompact; the converse 
statement that a 2-cocompact and non-elementary convergence action is geometrically finite is shown in [11, and its proof does not request the metrisability of the space $X=\Lambda G$.

(3) As a corollary we see that for each $L>0$ the set of $L$-uniformly conical points is $G$-invariant, although this is not clear at all from the dynamical definition.

Corollary 2.13. Let $\epsilon=\epsilon(1)>0$ given by Lemma 2.10.1. For any $R, L>0$, an $(\epsilon, R, L)$-transitional geodesic ray ends at a uniformly conical point $\xi \in \partial_{\lambda} G$.

As another consequence of the proof, we have the following result.

Corollary 2.14. Let $G \curvearrowright X$ be a geometrically finite action. Then there exists a constant $L>0$ such that for any conical point $\xi \in X$ there is a sequence of elements $g_{n} \in G$ such that for any geodesic $\gamma$ ending at $\xi$, we have

$$
\left[v, \xi\left[\gamma \subset \cup_{n \geq 1} B\left(g_{n}, L\right)\right.\right.
$$

for some $v \in \gamma$.

Remark. In the setting of Kleinian groups, this property is used to define uniformly conical points, cf. [24]. Here we do not need to assume that $G$ acts geometrically finitely on $\partial_{\lambda} G$. Also the corollary holds for "quasi-geodesics" instead of "geodesics".

We setup some notations for future discussions about uniformly conical points.

Let $\epsilon, R$ be given by Lemma 2.12. Denote by $\Lambda_{L}^{u c} G$ the set of uniformly conical points $\xi \in \Lambda G$ such that there exists an $(\epsilon, R, L)$-transitional geodesic ray $\gamma$ ending at $\xi$. It is obvious that $\Lambda_{L}^{u c} G$ is a $G$-invariant set.

Fixing a basepoint $o \in G$, denote by $\Lambda_{L, o}^{u c} G$ the set of all uniformly conical points $\xi \in \Lambda_{L}^{u c} G$ where a geodesic $\gamma$ between $o$ and $\xi$ is $(\epsilon, R, L)$-transitional.

Clearly, $G \cdot \Lambda_{L, o}^{u c} G=\Lambda_{L}^{u c} G$. Thus, the set $\Lambda_{L, o}^{u c} G$ can be thought as a fundamental domain for the action of $G$ on the set $\Lambda_{L}^{u c} G$.

Similarly, we define the set of uniformly conical points $\partial_{L, o}^{u c} G$ and $\partial_{L}^{u c} G$ on the Floyd boundary $\partial_{\lambda} G$. By Proposition 2.4 there exists one-to-one correspondence between $\Lambda_{L}^{u c} G$ and $\partial_{L}^{u c} G$.

2.5. Contracting property. Recall that $\|\cdot\|$ denotes the diameter of a set in a metric space.

Definition 2.15. For $c \geq 1$, a subset $X$ is called $c$-contracting in a metric space $Y$ if there exists $\mu_{c}, D_{c}>0$ such that the following holds

$$
\left\|\operatorname{Proj}_{X}(\gamma)\right\|<D_{c}
$$

for any $c$-quasi-geodesic $\gamma$ in $Y$ with $N_{\mu_{c}}(X) \cap \gamma=\emptyset$.

A collection of $c$-contracting subsets is referred to as a c-contracting system if $\mu_{c}, D_{c}$ depends only on $c$.

A system $\mathbb{X}$ has a bounded intersection property if for any $\epsilon>0$ there exists $\mathcal{R}=\mathcal{R}(\epsilon)>0$ such that

for any two distinct $X, X^{\prime} \in \mathbb{X}$.

$$
\left\|N_{\epsilon}(X) \cap N_{\epsilon}\left(X^{\prime}\right)\right\|<\mathcal{R}
$$

In what follows, our discussion applies to the Cayley graph of a relatively hyperbolic group $(G, \mathcal{P})$ with a finite generating set $S$. In particular, we are interested in the contracting system with bounded intersection given by the following lemma. 
Lemma 2.16. 13] Let $\mathbb{P}=\{g P: g \in G, P \in \tilde{\mathcal{P}}\}$, where $\tilde{\mathcal{P}}$ is a complete set of conjugacy representatives in $\mathcal{P}$. There exists $\mathcal{R}: \mathbb{R}_{>0} \rightarrow \mathbb{R}_{>0}$ such that the collection $\mathbb{P}$ is a c-contracting system with the $\mathcal{R}$-bounded intersection for each $c \geq 1$.

Proof. The contracting property is proven in [13, Proposition 8.5], and the bounded intersection is in [13, Corollary 5.7].

The following lemma will be often used further on.

Lemma 2.17. Let $\mathbb{P}$ be the collection of horospheres in (2.16). For any $c \geq 1$, there exist $\epsilon_{c}=\epsilon(c)>0$ such that for every c-quasi-geodesic $\gamma$ in $\mathscr{G}(G, S)$ and $\epsilon \geq \epsilon_{c}$ we have:

$\forall R \geq 0, \exists L=L(\epsilon, R)>0$ such that the condition $\max \left\{d\left(\gamma_{-}, X\right), d\left(\gamma_{+}, X\right)\right\}<$ $\epsilon$, for some $X \in \mathbb{P}$, implies that every point $z \in \gamma$ satisfying $d\left(z, \gamma_{-}\right), d\left(z, \gamma_{+}\right)>L$ is $\left(\epsilon_{c}, R\right)$-deep in $X$.

Proof. The result is proved in [27, Lemma 2.8] for geodesics. We provide below a proof to precise the choice of the constants.

By Lemma 2.16, let $\mu_{c}, D_{c}$ be the constants such that for any $X \in \mathbb{P}$, for any $c$ quasi-geodesic outside $N_{\mu_{c}}(X)$, the diameter of its projection to $X$ is upper bounded by $D_{c}$.

Set $\epsilon_{c}:=c\left(2 \mu_{c}+D_{c}\right)+c$. If a $c$-quasi-geodesic has two endpoints in $N_{\mu_{c}}(X)$ for $X \in \mathbb{P}$, then it lies in $N_{\epsilon_{c}}(X)$. Indeed, if $x, y \in \gamma$ satisfy

$$
\max \{d(x, X), d(y, X)\} \leq \mu_{c}
$$

and $] x, y\left[\gamma \cap N_{\mu_{c}}(X)=\emptyset\right.$, then by Lemma 2.16, $d(x, y) \leq 2 \mu_{c}+D_{c}$. Since $\gamma$ is $c$-quasi-geodesic we have $\ell\left([x, y]_{\gamma}\right) \leq \epsilon_{c}$ and $[x, y]_{\gamma} \subset N_{\epsilon_{c}}(X)$.

Set $L=c\left(2 \epsilon+D_{c}\right)+c+R$ for $\epsilon \geq \epsilon_{c}$. We first claim $\gamma \cap N_{\mu_{c}}(X) \neq \emptyset$. Otherwise, we obtain using projection the following

$$
2 L \leq \ell(\gamma) \leq c d\left(\gamma_{-}, \gamma_{+}\right)+c \leq c\left(2 \epsilon+D_{c}\right)+c .
$$

This gives a contradiction by the choice of $L$. Thus, there exist the entry point $x$ and the exit point $y$ of $\gamma$ in $N_{\mu_{c}}(X)$.

By the same argument one obtains

$$
\max \left\{\ell\left(\left[\gamma_{-}, x\right]_{\gamma}\right), \ell\left(\left[y, \gamma_{+}\right]_{\gamma}\right)\right\} \leq c\left(\epsilon+\mu_{c}+D_{c}\right)+c<L .
$$

Since $\min \left\{d\left(z, \gamma_{-}\right), d\left(z, \gamma_{+}\right)\right\}>L$, we have $z \in[x, y]_{\gamma}$. Then we obtain

$$
\min \{d(x, z), d(z, y)\} \geq L-\left(\epsilon+\mu_{c}+D_{c}\right)>R .
$$

By definition of $\epsilon_{c}$, we have $[x, z]_{\gamma} \subset N_{\epsilon_{c}}(X)$ and $[z, y]_{\gamma} \subset N_{\epsilon_{c}}(X)$. So $z$ is $\left(\epsilon_{c}, R\right)$-deep in $X$.

Remark. By the proof, we actually have $\epsilon_{c}>\mu_{c}$, where $\mu_{c}$ is uniform for every $X \in \mathbb{P}$ by Lemma 2.16 .

In what follows, we take constants $\epsilon, R$ as in Convention 2.18

Convention 2.18 (About $\epsilon_{c}, R_{c}$ ). When talking about $\left(\epsilon_{c}, R_{c}, L\right)$-transitional c-quasigeodesics, or $\left(\epsilon_{c}, R_{c}\right)$-transitional and $\left(\epsilon_{c}, R_{c}\right)$-deep points in a c-quasi-geodesic, we assume without explicitely specifying the quantifiers:

(1) $\epsilon_{c}=\epsilon(c)>\mu_{c}$ to satisfy Lemmas 2.10 and 2.17, where $\mu_{c}$ is given by Definition 2.15.

(2) $R_{c}>\mathcal{R}(\epsilon)$, where $\mathcal{R}$ is given by Lemma 2.16. 
Besides the peripheral cosets (horospheres), transitional quasi-geodesics provide another source of contracting subsets.

Lemma 2.19 (Transitional geodesic is contracting). For any $L \geq 0$, any $(\epsilon, R, L)$ transitional geodesic $\gamma$ is 1-contracting.

Remark. The same argument also applies (with natural changes for the constants) to show that if $\gamma$ is $c$-quasi-geodesic then it is contracting for any $c \geq 1$. For our purposes, we only need to consider the case when $c=1$.

Proof. Let $\kappa=\kappa(\epsilon, R)$ given by Lemma 2.10 and $\phi$ given by Lemma 2.6. By Lemma 2.6 , there exists $D_{0}=\phi(\kappa / 4)>0$ such that for any $v \in G$, a geodesic segment outside the ball $B\left(v, D_{0}\right)$ has $\mathfrak{l}_{v}$-Floyd length less than $\kappa / 4$.

Let $D=2\left(L+2 D_{0}+1\right)$ and $\mu=\phi(\kappa / 2)$. Let $\beta$ be a geodesic such that $\beta \cap N_{\mu}(\gamma)=\emptyset$. Let $x, y \in \mathbf{P r o j}_{\gamma}(\beta)$ such that $d(x, y)=\left\|\mathbf{P r o j}_{\gamma}(\beta)\right\|$. We are going to prove that $d(x, y) \leq D$. Suppose by contradiction that $d(x, y)>D$.

Assume that $x, y$ are projection points of $\tilde{x}, \tilde{y} \in \beta$ respectively. Observe that

$$
2 d(z,[x, \tilde{x}]) \geq d(z, x), 2 d(z,[y, \tilde{y}]) \geq d(z, y),
$$

for any $z \in[x, y]_{\gamma}$. We only prove the first inequality; the second one is completely analogous. Let $m \in[x, \tilde{x}]$ such that $d(z, m)=d(z,[x, \tilde{x}])$. Note that $d(m, z)+$ $d(m, \tilde{x}) \geq d(x, \tilde{x})$ by the shortest point property. Since $d(x, \tilde{x})=d(x, m)+d(m, \tilde{x})$ we obtain $d(m, z) \geq d(x, m)$. Then $d(z, x) \leq d(z, m)+d(m, x) \leq 2 d(z, m)$ implying (8).

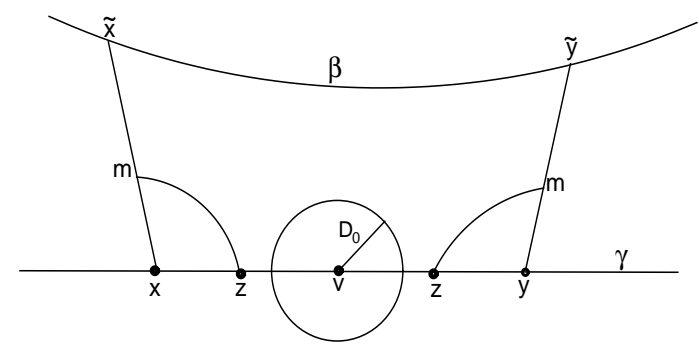

Since $d(x, y)>D$, there exists $z \in[x, y]_{\gamma}$ such that

$$
\min \{d(z, x), d(z, y))>D / 2=L+2 D_{0}+1 .
$$

Since $\gamma$ is $(\epsilon, R, L)$-transitional, one of the intervals $[x, z]_{\gamma}$ or $[z, y]_{\gamma}$ contains an $(\epsilon, R)$-transitional point $v$ such that $\min \{d(x, v), d(y, v)\}>2 D_{0}$. Hence by (8), $\min \{d(v,[x, \tilde{x}]), d(v,[y, \tilde{y}]))\}>D_{0}$. By the choice of $D_{0}=\phi(\kappa / 4)$, we have

$$
\max \left\{\rho_{v}(x, \tilde{x}), \rho_{v}(y, \tilde{y})\right\}<\kappa / 4 \text {. }
$$

From the other hand, $v$ is $(\epsilon, R)$-transitional, so $\rho_{v}(x, y) \geq \kappa$ by Lemma 2.10 . Hence, $\rho_{v}(\tilde{x}, \tilde{y})>\kappa / 2$ and thus $d(v, \beta) \leq \mu$ which is impossible.

For a c-quasigeodesic we denote by $\epsilon_{c}=\epsilon(c), R_{c}=\mathcal{R}\left(\epsilon_{c}\right)$ any numbers satisfying Convention 2.18 (in particular $\epsilon_{1}$ and $R_{1}$ correspond to geodesics). In the following Proposition we will establish a "thinness" of a triangle whose two sides are transitional geodesics. 
Proposition 2.20 (Transitional triangle is thin). For any $L, c>0$ and the there exist constants $D=D(c), M=M(L, c), L^{\prime}=L^{\prime}(L, c)>0$ with the following properties.

Let $\alpha_{1}, \alpha_{2}$ be $\left(\epsilon_{1}, R_{1}, L\right)$-transitional geodesic rays issuing at o and ending at $\xi \neq \eta \in \Lambda G$ respectively. Then for any c-quasi-geodesic $\gamma$ with $\gamma_{-} \in \alpha_{1}, \gamma_{+} \in \alpha_{2}$, the following holds.

(1) $\gamma$ is $\left(\epsilon_{c}, R_{c}, L^{\prime}\right)$-transitional.

(2) If the length of $\gamma$ is sufficiently large then there exists an $\left(\epsilon_{c}, R_{c}\right)$-transitional point $z \in \gamma$ such that $d\left(z, \alpha_{1} \cup \alpha_{2}\right) \leq D$ and $d\left(z, \alpha_{i}\right) \leq M$ for $i=1,2$.

(3) Let $d(o,[\xi, \eta])$ denote the distance from o to a geodesic between $\xi, \eta$. If $\min \left\{d\left(\gamma_{-}, o\right), d\left(\gamma_{+}, o\right)\right\} \gg 0$. Then $|d(o,[\xi, \eta])-d(o, \gamma)| \leq M$.

Remark. Note that in (2) $D$ is a uniform constant not depending on $L$, this will play a crucial role in establishing Lemma 5.13 below.

Proof. Let $\kappa=\kappa\left(\epsilon_{c}, R_{c}\right)$ given by Lemma 2.10 and $D=\phi(\kappa / 2)$, where $\phi$ is given by Lemma 2.6. The constant $L^{\prime}$ will be computed below.

(1) Given a point $x$ in $\gamma$, assume that $x$ is $\left(\epsilon, R_{c}\right)$-deep in some $X \in \mathbb{P}$. Let $x_{-}, x_{+}$be the entry and exit points of $\gamma$ in $N_{\epsilon_{c}}(X)$ respectively.

Observe first that $x_{-}, x_{+}$are $\left(\epsilon_{c}, R_{c}\right)$-transitional in $\gamma$. Indeed, if not, there exists $Y \in \mathbb{P}$ such that $x_{-}$is $\left(\epsilon_{c}, R_{c}\right)$-deep in $Y$. Then $Y \neq X$ by the choice of $x_{-}$as the entry point of $\gamma$ in $N_{\epsilon_{c}}(X)$. Since $d\left(x, x_{-}\right) \geq R_{c}$, we have $\left\|N_{\epsilon}(X) \cap N_{\epsilon}(Y)\right\| \geq$ $R_{c}>\mathcal{R}\left(\epsilon_{c}\right)$ by Convention 2.18. This contradicts to Lemma 2.16 .

To find a constant $L^{\prime}$ we will check when the opposite inequality:

$$
\min \left\{\ell\left(\left[x, x_{-}\right]_{\gamma}\right), \ell\left(\left[x, x_{+}\right]_{\gamma}\right)\right\}>L^{\prime}
$$

is not valid. We have $\ell\left(\left[x_{-}, x_{+}\right]_{\gamma}\right) \geq 2 L^{\prime}$. Since $x_{-}, x_{+}$are $\left(\epsilon_{c}, R_{c}\right)$-transitional, by Lemma 2.10 .

$$
\min \left\{\rho_{x_{-}}\left(\gamma_{-}, \gamma_{+}\right), \rho_{x_{+}}\left(\gamma_{-}, \gamma_{+}\right)\right\}>\kappa
$$

By the triangle inequality

$$
\max \left\{\rho_{x_{-}}\left(\gamma_{-}, o\right), \rho_{x_{-}}\left(o, \gamma_{+}\right)\right\} \geq \frac{\kappa}{2},
$$

and the same for $\rho_{x_{+}}$. Then $\max \left\{d\left(x_{-}, \alpha_{1} \cup \alpha_{2}\right), d\left(x_{+}, \alpha_{1} \cup \alpha_{2}\right)\right\} \leq D=\phi(\kappa / 2)$. For concreteness consider the case that

$$
d\left(x_{-}, \alpha_{1}\right), d\left(x_{+}, \alpha_{2}\right) \leq D
$$

the other cases are similar and even easier.

Project $x_{-}, x_{+}$to $x_{-}^{\prime}, x_{+}^{\prime} \in X$ so that $d\left(x_{-}, x_{-}^{\prime}\right), d\left(x_{+}, x_{+}^{\prime}\right) \leq \epsilon_{c}$. So

$$
d\left(x_{-}^{\prime}, \alpha_{1}\right), d\left(x_{+}^{\prime}, \alpha_{2}\right) \leq \epsilon_{c}+D
$$

and $N_{D+\epsilon_{c}}(X) \cap \alpha_{i} \neq \emptyset(i=1,2)$.

Let $w \in X$ be a projection point of $o$ to $X$. We claim that

$$
d\left(w, \alpha_{i}\right) \leq D_{2}:=\max \left\{D+\epsilon_{c}+D_{1}, \mu_{1}+D_{1}\right\}(i=1,2),
$$

where $\mu_{1}, D_{1}>0$ are given for 1-contracting $X \in \mathbb{P}$ such that (7) holds.

Indeed if, first, $o \in N_{\mu_{1}}(X)$ then there is nothing to prove. If not, there are two more cases: if $\alpha_{i} \cap N_{\mu_{1}}(X)=\emptyset$, then by the contracting property we have $d\left(w, \alpha_{i}\right) \leq$ $D+\epsilon_{c}+D_{1}$; otherwise the projection on $X$ of the maximal connected subcurve of $\gamma$, situated outside of $N_{\mu_{1}}(X)$ and containing $o$, gives $d\left(w, \alpha_{i}\right) \leq \mu_{1}+D_{1}$. So 11 follows. 
Let $L_{0}=L\left(\epsilon_{c}+D+D_{2}, R_{1}+L\right)$ given by Lemma 2.17. Set

$$
L^{\prime}=2 c\left(D_{2}+D+L_{0}+2 \epsilon_{c}\right)+c^{2} .
$$

Since $\gamma$ is a $c$-quasi-geodesic, we have

$$
\begin{aligned}
d\left(x_{-}^{\prime}, x_{+}^{\prime}\right) \geq \ell\left(\left[x_{-}, x_{+}\right]_{\gamma}\right) / c-c-2 \epsilon_{c} & \geq 2 L^{\prime} / c-c-2 \epsilon_{c} \\
& \geq 4\left(\epsilon_{c}+D+D_{2}+L_{0}\right) .
\end{aligned}
$$

Since $\max \left\{d\left(x_{-}^{\prime}, \alpha_{1}\right), d\left(x_{+}^{\prime}, \alpha_{2}\right)\right\} \leq \epsilon_{c}+D$, we obtain from (11)

$$
\left\|\alpha_{1} \cap N_{\epsilon_{c}+D+D_{2}}(X)\right\| \geq d\left(x_{-}^{\prime}, w\right),\left\|\alpha_{2} \cap N_{\epsilon_{c}+D+D_{2}}(X)\right\| \geq d\left(x_{+}^{\prime}, w\right) .
$$

We have $d\left(x_{-}^{\prime}, w\right)+d\left(x_{+}^{\prime}, w\right) \geq d\left(x_{-}^{\prime}, x_{+}^{\prime}\right) \geq 4\left(\epsilon_{c}+D+D_{2}+L_{0}\right)$. Thus

$$
\max _{i=1,2}\left\|\alpha_{i} \cap N_{\epsilon_{c}+D+D_{2}}(X)\right\| \geq 2\left(\epsilon_{c}+D+D_{2}+L_{0}\right) .
$$

Hence, $\alpha_{i}$ contains a subcurve of length at least $2 L_{0}$ such that its endpoints lie in $N_{\epsilon_{c}+D+D_{2}}(X)$. By the choice of $L_{0}$ and Lemma 2.17 $\alpha_{i}$ contains an $\left(\epsilon_{1}, R_{1}+L\right)$ deep point in $X$. This gives a contradiction, as $\alpha_{i}$ is $\left(\epsilon_{1}, R_{1}, L\right)$-transitional. So for the value of $L^{\prime}$ chosen in (12) the inequality (9) is not valid. The statement (1) is proved.

(2) By the statement (1) $\gamma$ is $\left(\epsilon_{c}, R_{c}, L^{\prime}\right)$-transitional. Lemma 2.19 implies that $\gamma$ is contracting. By the projection argument (used to prove (11)) we have a constant $D_{3}=D_{3}\left(\epsilon_{c}, R_{c}, L^{\prime}\right)>0$ such that for any projection point $v$ of $o$ to $\gamma$ we have $d\left(v, \alpha_{i}\right) \leq D_{3}$ for $i=1,2$.

Remark. We need a new constant $D_{3}$ (and not $D_{2}$ used above) since we project now on $\gamma$ and not on a horosphere.

Recall that $D=\phi(\kappa / 2)$. By Lemma 2.6, for any $z \in G$, a geodesic segment outside $B(z, D)$ has $\mathfrak{l}_{z}$-Floyd length less than $\kappa / 2$.

The curve $\gamma$ is quasigeodesic and its length is sufficently large. So by continuity of the distance function $d(v, x)(x \in \gamma)$ we find a point $z^{\prime}$ such that $D+D_{3}+L^{\prime} \leq$ $d\left(v, z^{\prime}\right) \leq D+D_{3}+L^{\prime}+1$. Since $\gamma$ is $\left(\epsilon_{c}, R_{c}, L^{\prime}\right)$-transitional by Definition 2.11 there exists an $\left(\epsilon_{c}, R_{c}\right)$-transitional point $z \in \gamma$ for which $d\left(z^{\prime}, z\right) \leq l\left(\left[z, z^{\prime}\right]_{\gamma}\right) \leq L^{\prime}$. We obtain

$$
D+D_{3} \leq d(v, z) \leq D+D_{3}+2 L^{\prime}+1
$$

Then

$$
d\left(z, \alpha_{i}\right) \leq d(z, v)+d\left(v, \alpha_{i}\right) \leq M
$$

where $M=2 L^{\prime}+2 D_{3}+D+1$.

To prove the first claim of (2) assume for definiteness that $z \in\left[v, \gamma_{+}\right]$. Lemma 2.10 yields $\rho_{z}\left(v, \gamma_{+}\right) \geq \kappa$.

Let $z_{2} \in \alpha_{2}$ such that $d\left(v, z_{2}\right) \leq D_{3}$. We have $d\left(z,\left[v, z_{2}\right]\right) \geq d(z, v)-d\left(z_{2}, v\right) \geq$ $D+D_{3}-D_{3}=D$. By Lemma $2.6 \rho_{z}\left(v, z_{2}\right)<\kappa / 2$ and so $\rho_{z}\left(z_{2}, \gamma_{+}\right) \geq \rho_{z}\left(v, \gamma_{+}\right)-$ $\rho_{z}\left(v, z_{2}\right) \geq \kappa / 2$. Lemma 2.6 gives

$$
d\left(z, \alpha_{2}\right) \leq D .
$$

The statement (2) is proved.

(3) Since $\xi, \eta$ are distinct, by Lemma 2.6, there exists $n_{0}=n_{0}(\xi, \eta), r=r(\xi, \eta)>$ 0 such that if

$$
\min \left\{d\left(\gamma_{-}, o\right), d\left(\gamma_{+}, o\right)\right\}>n_{0},
$$

then $d(o, \gamma) \leq r$. In the proof of the statement (2), we projected $o$ to a point $v$ in $\gamma$, and found an $(\epsilon, R)$-transitional point $z \in \gamma$ such that $d(v, z) \leq M$. 
Since $d(o, z) \leq M+r$ (and these constants do not depend on $\gamma$ ) up to increasing $n_{0}$, by Lemma 2.6 we have

$$
\max \left\{\bar{\rho}_{z}\left(\xi, \gamma_{-}\right), \bar{\rho}_{z}\left(\eta, \gamma_{+}\right)\right\} \leq \kappa / 4
$$

The point $z$ is $(\epsilon, R)$-transitional, thus $\bar{\rho}_{z}\left(\gamma_{-}, \gamma_{+}\right) \geq \kappa$, and so $\bar{\rho}_{z}(\xi, \eta) \geq \kappa / 2$. Consequently $d(z,[\xi, \eta]) \leq D$ which yields:

$$
\begin{aligned}
d(o,[\xi, \eta]) & \leq d(o, z)+d(z,[\xi, \eta]) \leq d(o, \gamma)+M+d(z,[\xi, \eta]) \\
& \leq d(o, \gamma)+M+D .
\end{aligned}
$$

By symmetry, we obtain $d(o, \gamma) \leq d(o,[\xi, \eta])+M+D$.

Since $D$ is a uniform constant not depending on $L$ we put $M:=M+D$. Then the statements of (2) and (3) are both valid for the same constant $M$. The Proposition is proved.

The claim (3) of the Proposition and Lemma 2.12 imply:

Corollary 2.21. Suppose $(G, \mathcal{P})$ is a relatively hyperbolic pair. Then for any $L>0$, there exists $M=M(L)$ such that for any $\xi, \eta \in \Lambda_{L, o}^{u c} G$ or $\xi, \eta \in \partial_{L, o}^{u c} G$, the distance $d(o,[\xi, \eta])$ is comparable with the distance $d(o, \gamma)$ where $\gamma$ is a c-quasi-geodesic with the endpoints on the corresponding geodesic rays converging to $\xi$ and $\eta$..

\section{Patterson-Sullivan measures on ends of a Geodesic tree}

In this section, we shall construct an iterated transitional tree having several nice properties which will allow us to carry out the Patterson's construction on this tree. The space of ends of the tree equipped with the Patterson-Sullivan measure will give rise to an Ahlfors regular subset of the boundary.

3.1. Iterated Transitional Trees. Let $(G, \mathcal{P})$ be a relatively hyperbolic pair and $\mathscr{G}(G, S)$ the Cayley graph of $G$ with respect to $S$. The existence of large transitional trees is established in [27, Theorem 5.9]. The main difference of the construction below is that these trees will be equipped with certain periodicity. By this reason we call them iterated transitional trees. We start by recalling several results from [27.

Definition 3.1 (Partial Cone). For $\epsilon, R \geq 0$, the partial cone $\Omega_{\epsilon, R}(g)$ at $g \in G$ is the set of elements $h \in G$ such that there exists a geodesic $\gamma=[1, h]$ containing $g$ and one of the following holds.

(1) $d(1, h) \leq d(1, g)+2 R$,

(2) $\gamma$ contains an $(\epsilon, R)$-transitional point $v$ such that $d(v, g) \leq 2 R$.

For $\Delta \geq 0, n \geq 0$, define

$$
A(g, n, \Delta)=\{h \in G: n-\Delta \leq d(1, h)-d(1, g)<n+\Delta\},
$$

for any $g \in G$. For simplicity we write $A(n, \Delta):=A(1, n, \Delta)$. For $r, \epsilon, R, \Delta>0$, define

for any $g \in G, n \geq 0$.

$$
\Omega_{\epsilon, R}(g, n, \Delta)=\Omega_{\epsilon, R}(g) \cap A(g, n, \Delta),
$$

For fixed $\epsilon, R>0$, two partial cones $\Omega_{\epsilon, R}(g), \Omega_{\epsilon, R}\left(g^{\prime}\right)$ are of same type if

$$
g^{\prime} g^{-1} \cdot \Omega_{\epsilon, R}(g)=\Omega_{\epsilon, R}\left(g^{\prime}\right) .
$$

By abuse of language, we say that $g, g^{\prime}$ have the same partial cone types.

The following result generalizes the result of Cannon [5] for hyperbolic groups. 
Lemma 3.2 (Finiteness of partial cone types). [27, Lemma B.1] There exist $\epsilon, R_{0}>0$ such that for any $R>R_{0}$, there are at most $M=M(\epsilon, R)$ types among all $(\epsilon, R)$ partial cones $\left\{\Omega_{\epsilon, R}(g): g \in G\right\}$.

The following is a key technical result in [27, Lemma 5.8].

Lemma 3.3. There exist $\epsilon, R, \Delta, \theta, L_{0}>0$ with the following property.

For any $L>L_{0}$ there exists a subset $\hat{G}$ of $G$ such that

$$
\sharp\left(\Omega_{\epsilon, R}(g, L, \Delta) \cap \hat{G}\right)>\theta \cdot \exp \left(L \cdot \delta_{G, S}\right), 1 \in \hat{G}
$$

for any $g \in \hat{G}$.

Convention $3.4(\epsilon, R, \Delta)$. Until the end of Section 3, the constants $\epsilon, R, \Delta>0$ are given by Lemmas 3.2 and 3.3, and satisfy Convention 2.18.

The following terminology comes from the paper [1] which was certainly very motivating for us.

Definition 3.5 (Iterated Tree Set). For given $L>0$, an $L$-iterated tree set $T$ in $G$ is a union of a sequence of sets $T_{i}(i \geq 0)$ in $G$ defined inductively as follows.

Let $T_{0}=\{1\}$. Assume that $T_{i}$ is defined for $i \geq 0$. The children $T(x)$ of $x \in T_{i}$ is a subset in $\Omega_{\epsilon, R}(x, L, \Delta)$. Then $T_{i+1}$ is the union of children of all $x \in T_{i}$.

Recall that a subset $Z$ of a metric space space $(X, d)$ is called $C$-separated if the distanced $\left(z_{1}, z_{2}\right) \geq C$ for every pair of distinct points $\left\{z_{1}, z_{2}\right\} \subset Z$. The following fact is elementary.

Lemma 3.6. Let $(X, d)$ be a proper metric space on which a group $G \subset \operatorname{Isom}(X)$ acts properly on $X$. For any orbit $G o(o \in X)$ and $C>0$ there exists a constant $\theta=\theta(G o, C)>0$ with the following property.

For any finite set $Y$ in Go, there exists a $C$-separated subset $Z \subset Y$ such that $\sharp Z \geq \theta \cdot \sharp Y$.

Proof. Let $Z$ be a maximal $C$-separated set in $Y$. We have $Y \subset N_{C}(Z)$. Since the action of $G$ on $(X, d)$ is proper, any ball of radius $C$ contains at most $N$ points in Go. The result follows for $\theta:=1 / N$.

An $(\epsilon, R, L)$-transitional geodesic tree $\mathcal{T}$ rooted at $o$ in $\mathscr{G}(G, S)$ is a tree subgraph with a distinguished vertex $x$ such that every branch in $\mathcal{T}$ originating at $x$ is a $(\epsilon, R, L)$-transitional geodesic in $\mathscr{G}(G, S)$.

In order to obtain a useful theory of Patterson-Sullivan measures, certain symmetry on the iterated tree set is required. This is the content of the following.

Lemma 3.7 (Existence of iterated transitional trees). There exist constants $L_{0}, C_{0}, t_{0}, n_{0}>$ 0 such that for $L>L_{0}, C>C_{0}$ there are $\theta=\theta(C)$ and $L^{\prime}=L^{\prime}(L)$ and an iterated tree set $T$ parameterized by $(\epsilon, R, L)$ with the following properties:

(1) $x^{-1} T(x)=y^{-1} T(y)$ for any $x \in T_{t}, y \in T_{t+n_{0}}$ and $t \geq t_{0}$,

(2) $x^{-1} T(x)=y^{-1} T(y)$ for any $x, y \in T_{t}$ and $t \geq t_{0}$.

(3) $\sharp T(x) \geq \theta \cdot \exp \left(\delta_{G, S} L\right)$ for any $x \in T$.

(4) $T(x)$ is $C$-separated for any $x \in T$.

(5) there exists an $\left(\epsilon, R, L^{\prime}\right)$-transitional geodesic tree $\mathcal{T}$ rooted at 1 in $\mathscr{G}(G, S)$ such that the vertex set $\mathcal{T}^{0}$ contains $T$, and lies in $N_{L^{\prime}}(T)$. 
Proof. Set $L_{0}=\Delta$, and all other constants will be defined in the proof. We divide the proof into 3 steps for the reader convenience.

Step 1: At this step we construct the iterated tree set $T$ with properties (1-3). The construction proceeds by an induction argument. Set $T_{0}=\{1\}$ to start.

Let $M$ be the number of $(\epsilon, R)$-partial cone types in $G$ given by Lemma 3.2 , and $\hat{G}$ the set by Lemma 3.3 . Then there exists $T_{1} \subset \Omega_{\epsilon, R}(1, L, \Delta) \cap \hat{G}$ such that every element in $T_{1}$ has the same partial cone type and the inequality (14) holds for $g=1$ where the constant $\kappa$ is divided by $M$. By Lemma 3.6, we can also require that $T_{1}$ is $C$-separated, where $\kappa$ is further decreased and depends on $C$ (given in Step 3 below).

Fix some $x_{1} \in T_{1}$. Up to dividing $\theta$ by $M$ again, we choose $Y$ to be a subset of $\Omega_{\epsilon, R}\left(x_{1}, L, \Delta\right) \cap \hat{G}$ such that the inequality (14) holds for $Y$ and every element in $Y$ has the same partial cone type. By the same reason, we can choose $Y$ to be $C$-separated. Since all $x \in T_{1}$ are of same type as $x_{1}$, we could define

$$
T(x):=x x_{1}^{-1} Y \subset \Omega_{\epsilon, R}(x, L, \Delta) .
$$

Then all elements in the union $T_{2}:=\cup_{x \in T_{1}} T(x)$ have the same partial cone types. We note that $Y$ is chosen to be contained in $\hat{G}$, but $T_{2}$ may not be in $\hat{G}$.

We repeat the same argument to construct $T_{i}$ for $i \geq 3$, with a sequence of divisions of $\kappa$. By construction, all elements in the constructed $T_{i}$ are of the same partial cone type. Since there are finitely many partial cone types, we obtain that there are $1 \leq t_{0}, n_{0} \leq M$ so that $x^{-1} T(x)=y^{-1} T(y)$ for any $x \in T_{t}, y \in T_{t+n_{0}}$ and $t \geq t_{0}$. This also implies that the division of $\theta$ stops after at most $n_{0}$ times, and thus $\theta$ in the inequality (14) can be chosen uniformly for all $T(x)$ where $x \in T_{i}$ $(i \geq 1)$. The set $T$ satisfies the properties $(1-3)$.

Step 2: Using the iterated tree set $T$, we will now construct a geodesic graph $\mathcal{T}$.

Without loss of generality assume that $\kappa<1$. The root of $\mathcal{T}$ is $\mathcal{T}_{0}=\{1\}$. Assume that $\mathcal{T}_{i}$ is defined for $i \geq 0$ and for each terminal vertex $x \in \mathcal{T}_{i}$, denote by $\gamma_{x}$ the geodesic $[1, x]$ in $\mathcal{T}_{i}$. We choose a geodesic $[x, y]$ for each $y \in T(x)$. Since $T_{i}(x)$ is a subset in $\Omega_{\epsilon, R}(x, L, \Delta) \cap \hat{G}$, we set

$$
\mathcal{T}_{i+1}=\cup_{x \in \mathcal{T}_{i}}\left(\cup_{y \in T(x)} \gamma_{x} \cdot[x, y]\right),
$$

where $\gamma_{x} \cdot[x, y]$ is a geodesic in $\mathscr{G}(G, S)$. Inductively, we get the $\operatorname{limit} \mathcal{T}=\lim _{i \rightarrow \infty} \mathcal{T}_{i}$. By construction, each geodesic ray originating at 1 is $\left(\epsilon, R, L^{\prime}\right)$-transitional for $L^{\prime}:=$ $L+2 R+\Delta$. By construction we have $T \subset \mathcal{T}^{0} \subset N_{L^{\prime}}(T)$.

Step 3: We now prove that $\mathcal{T}$ is a geodesic tree rooted at 1 in $\mathscr{G}(G, S)$. Indeed, if not, there exist two distinct geodesics $\alpha_{1}, \alpha_{2}$ in $\mathcal{T}$ with the same endpoints $x, w \in T$ such that the length of $\alpha_{1}, \alpha_{2}$ is minimal among all such choices. Assume that $x$ is closer to 1 than $w$. Consider two points $y_{i} \in \alpha_{i} \cap T(x)$ for $i=1,2$. By the choice of $\alpha_{1}, \alpha_{2}$, we have $y_{1} \neq y_{2}$. Then by construction, $d\left(y_{i}, w\right) \geq L-\Delta$ for $i=1,2$. Moreover, there exists an $(\epsilon, R)$-transitional point $z_{1} \in \alpha_{1}$ such that $d\left(y_{1}, z_{1}\right) \leq 2 R$.

Let $D_{0}=\phi(\kappa)$, where $\phi$ is given by Lemma 2.6 and $\kappa=\kappa(\epsilon, R)$ by Lemma 2.10 . There exists $z_{2} \in \alpha_{2}$ such that $d\left(z_{1}, z_{2}\right) \leq D_{0}$ and then $d\left(y_{1}, z_{2}\right) \leq 2 R+D_{0}$. We can choose $\tilde{y}_{2} \in \alpha_{2}$ such that $d\left(x, \tilde{y}_{2}\right)=d\left(x, y_{1}\right)$. Hence $d\left(z_{2}, \tilde{y}_{2}\right)=\left|d\left(x, z_{2}\right)-d\left(x, \tilde{y}_{2}\right)\right|=$ $\left|d\left(x, z_{2}\right)-d\left(x, y_{1}\right)\right| \leq 2 R+D_{0}$. It follows that $d\left(y_{1}, \tilde{y}_{2}\right) \leq 2\left(2 R+D_{0}\right)$.

Since $y_{1}, y_{2}$ lie in the annulus $A(x, L, \Delta)$, we get $\left|d\left(x, y_{1}\right)-d\left(x, y_{2}\right)\right| \leq 2 \Delta$, and then $d\left(y_{2}, \tilde{y}_{2}\right)=\left|d\left(x, \tilde{y}_{2}\right)-d\left(x, y_{2}\right)\right| \leq 2 \Delta$. It follows that $d\left(y_{1}, y_{2}\right) \leq 2\left(2 R+D_{0}+\Delta\right)$. 
Choosing now the constant $C$ to be greater than

$$
C_{0}:=2(2 R+\phi(\kappa / 2)+\Delta)
$$

we obtain that $T(x)$ is $C$-separated in $\Omega_{\epsilon, R}(x, L, \Delta)$, and $d\left(y_{1}, y_{2}\right) \geq C_{0}>2(2 R+$ $\left.D_{0}+\Delta\right)$ which is a contradiction. Thus, $\mathcal{T}$ is a rooted geodesic tree.

Remarks. (1) By Lemma 2.12 the boundary of the tree $\mathcal{T}$ (in $\Lambda G$ or in $\partial_{\lambda} G$ ) constructed above consists of uniformly conical points.

(2) The constant $C_{0}$ in 16 is bigger than we really need in the above proof (it is enough to replace $\varphi(\kappa / 2)$ by the smaller term $\left.D_{0}=\varphi(\kappa)\right)$ ) but we do need such a constant in the next lemma.

In the next two lemmas, we shall derive more properties of the sets $T$ and $\mathcal{T}$ constructed in Lemma 3.7. To this end, we recall the notion of Poincaré series.

For a subset $X \subset G$ and a point $o \in G$, set

$$
\Theta_{X}(s, o)=\sum_{g \in X} \exp (-s d(o, g)), s \geq 0 .
$$

Define the critical exponent of $\Theta_{X}(s, o)$ to be

$$
\delta_{X, S}=\limsup _{n \rightarrow \infty} \frac{\log \sharp(B(o, n) \cap X)}{n},
$$

where $S$ is a fixed finite symmetric generating set of $G$, and $B(o, n)$ is the ball in the word metric of radius $n$ centered at $o$.

It is elementary fact that $\Theta_{X}(s, o)$ converges for $s>\delta_{X, S}$, and diverges for $s<\delta_{X, S}$.

Recall that the bilipschitz equivalence $\asymp_{\text {const }}$ between two functions means that they are comparable up to a constant (see Section 2). We have the following.

Lemma 3.8. Under the same assumptions as in Lemma 3.7, we have

$$
\Theta_{T}(s, x) \asymp{ }_{L} \Theta_{T}(s, y)
$$

for any $x, y \in T$ and $s \geq 0$, whenever one of the series converges.

Proof. Let $\Omega(x)$ be a cone at $x \in T$, which is the union of $y \in T$ such that the unique geodesic $[1, y]$ in the geodesic tree $\mathcal{T}$ contains $x$.

Claim. The Poincaré series of $T$ is bilipschitz equivalent to that of any cone at a vertex in $T$ :

$$
\Theta_{T}(s, 1) \asymp_{L} \Theta_{\Omega(x)}(s, x),
$$

for any $x \in T$.

Proof of the Claim. It follows from Lemma 3.7.(1) that after a finite time $t_{0}$, the set $T$ is periodic with a fixed period $n_{0}$. So it is enough to show 18 for $x \in T$ such that $t_{0} \leq d(1, x) \leq n_{0}+t_{0}$. By Lemma 3.7 2 , the cones based at points $y \in T_{t}$ have the same type where $t=d(1, x)$. Thus the number $a_{n}$ of points in $T$ situated at the distance $n$ from 1 is at most $C \cdot b_{n, x}$. Here $C$ is the number of elements in the ball $B(1, t)$, and $b(n, x)$ is the number of elements of $\Omega(x)$ at the distance $n$ from $x$. The same argument works in the opposite sense. The Claim follows. 
To complete the proof of the lemma, by $(18)$, it suffices to establish the following

$$
\Theta_{T}(s, 1) \asymp_{L} \Theta_{T \backslash \Omega(x)}(s, x),
$$

as 18 and 19 would imply $\Theta_{T}(s, 1) \asymp_{L} \Theta_{T}(s, x), \forall x \in T$.

For $y \in T \backslash \Omega(x)$, let $o$ be the farrest point to 1 such that $o \in T$ and $[1, o] \subset$ $[1, x] \cap[1, y]$, where the geodesics $[1, x]$ and $[1, y]$ are in the geodesic tree $\mathcal{T}$. The point $o$ will be referred to as the branch point of $[1, x]$ and $[1, y]$.

By Lemma 3.7. $[1, x],[1, y]$ are $\left(\epsilon, R, L^{\prime}\right)$-transitional geodesics, where $L^{\prime}=L^{\prime}(L)$. By Proposition $2.20,[x, y]$ is transitional and so is contracting by Lemma 2.19 .

Claim. There exists a uniform constant $D=D(L)>0$ such that $d(o,[x, y]) \leq D$.

Proof of the Claim. Let $z \in[x, y]$ be the projection of $o$ to a geodesic $[x, y]$ in the Cayley graph $\mathscr{G}(G, S)$. By the contracting property of $[x, y]$ it follows from the inequality 11 that there exists $D_{1}=D_{1}\left(\epsilon, R, L^{\prime}\right)$ such that

$$
\max \{d(z,[o, x]), d(z,[o, y])\} \leq D_{1} .
$$

So, let $x_{1} \in[o, x], y_{1} \in[o, y]$ such that $d\left(z, x_{1}\right) \leq D_{1}$ and $d\left(z, y_{1}\right) \leq D_{1}$.

Set $d(o, z)=d$, then

$$
\min \left\{d\left(o, x_{1}\right), d\left(o, y_{1}\right)\right\} \geq d-D_{1}
$$

Let $w \in\left[o, x_{1}\right] \cap T(o)$ where $T(o) \subset \Omega_{\epsilon, R}(o, L, \Delta)$. Then $d(o, w)<L+\Delta$. Furthermore since $x \in T$ there exists an $(\epsilon, R)$-transitional point $x_{2} \in[o, x]$ such that $d\left(w, x_{2}\right) \leq 2 R$, and so $d\left(o, x_{2}\right) \leq L+\Delta+2 R$. Using 20 we deduce

$$
d\left(x_{2},\left[x_{1}, y_{1}\right]\right) \geq d\left(x_{1}, o\right)-d\left(x_{2}, o\right)-2 D_{1} \geq K,
$$

where $K=d-3 D_{1}-L-\Delta-2 R$.

We affirm that

$$
K \leq \phi(\kappa / 2),
$$

where $\kappa$ and $\phi$ are universal constants given by Lemmas 2.10 and 2.6 respectively. Indeed, suppose 222 is not true, then $d\left(x_{2},\left[x_{1}, y_{1}\right]\right) \geq \phi(\kappa / 2)$. By Lemma 2.6 we have $\rho_{x_{2}}\left(x_{1}, y_{1}\right) \leq \kappa / 2$. Since $x_{2}$ is transitional, Lemma 2.10 yields $\rho_{x_{2}}\left(o, x_{1}\right) \geq \kappa$. It follows $\rho_{x_{2}}\left(o, y_{1}\right) \geq \kappa / 2$, and thus $\exists \tilde{x}_{2} \in[o, y]: d\left(x_{2},[o, y]\right)=d\left(x_{2}, \tilde{x}_{2}\right) \leq$ $\phi(\kappa / 2)$.

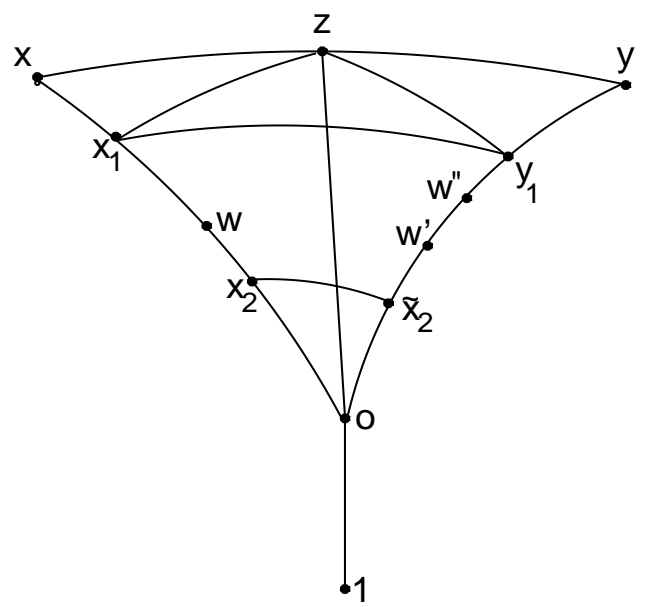


Following the argument of Step (3) of Lemma 3.7 we choose a vertex $w^{\prime} \in$ $[o, y]$ such that $d\left(o, w^{\prime}\right)=d(o, w)$. Then we have $d\left(\tilde{x}_{2}, w^{\prime}\right)=\left|d\left(o, \tilde{x}_{2}\right)-d(o, w)\right| \leq$ $d\left(\tilde{x}_{2}, w\right) \leq 2 R+\phi(\kappa / 2)$. Then $d\left(w^{\prime}, w\right) \leq d\left(w^{\prime}, \tilde{x}_{2}\right)+d\left(\tilde{x}_{2}, w\right) \leq 2(2 R+\phi(\kappa / 2))$. Let $w^{\prime \prime} \in T(o) \cap[o, y]$. Since $w \in \Omega_{\epsilon, R}(o, L, \Delta)$ we have $d\left(w^{\prime \prime}, w^{\prime}\right) \leq \mid d\left(o, w^{\prime}\right)-$ $d\left(o, w^{\prime \prime}\right) \mid \leq 2 \Delta$. Indeed $\left|\left(d\left(o, w^{\prime}\right)=d(o, w)\right)-L\right| \leq \Delta$ and also $\left|d\left(o, w^{\prime \prime}\right)-L\right| \leq \Delta$. Therefore for the vertices $w^{\prime \prime}, w \in T(o) \subset T$ we have $d\left(w, w^{\prime \prime}\right) \leq 2(\phi(\kappa / 2)+R+\Delta)$. This is impossible by $(16)$. The obtained contradiction implies that $K \leq \phi(\kappa / 2)$ and by definition of $K$ (see $(21))$, we have

$$
d(o, z)=d \leq D=D_{1}+\phi(\kappa / 2)+L+\Delta+2 R .
$$

The claim is proved.

The second claim implies

$$
d(o, x)+d(o, y) \geq d(x, y) \geq d(o, x)+d(o, y)-2 D \text {. }
$$

Given $o \in[1, x) \cap T$, we denote by $Y_{o}$ the set of elements $y \in T \backslash \Omega(x)$ such that $o \in[1, x]$ is the branch point of $[1, y]$ and $[1, x]$ in $\mathcal{T}$. The argument of the first Claim also yields

$$
\Theta_{\Omega(o)}(s, o) \asymp_{L} \Theta_{Y_{o}}(s, o)
$$

Then 18 and 23 imply

$$
\sum_{y \in Y_{o}} \exp (-s d(x, y)) \asymp_{L} \exp (-s d(o, x)) \cdot \Theta_{T}(s, 1),
$$

for every $o \in[1, x) \cap T$. By construction of $T$ in Lemma 3.7 the sequence of points $[1, x) \cap T$ has the property that any two consecutive points has a distance between $L-\Delta$ and $L+\Delta$. Summing up over all $o \in[1, x) \cap T$, we get

$$
\sum_{y \in T \backslash \Omega(x)} \exp (-s d(x, y)) \asymp_{L} \sum_{0 \leq k<d(1, x)} \exp (-s k) \cdot \Theta_{T}(s, 1) \asymp_{L} \Theta_{T}(s, 1),
$$

which proves 19 . The Lemma is proved.

Lemma 3.9. Under the same assumptions in Lemma 3.7, the Poincaré series $\Theta_{T}(s, 1)$ is divergent at $s=\delta_{T, S}$. Furthermore, $\lim _{L \rightarrow \infty} \delta_{T, S}=\delta_{G, S}$.

Proof. It is inspired by the proof of Proposition 4.1 in [8]. Consider the annulus set in $T$,

$$
A_{T}\left(g, n, 3 \Delta_{0}\right):=A\left(g, n, 3 \Delta_{0}\right) \cap T,
$$

where $\Delta_{0}:=\Delta+L+2 R$ and $n \geq 0$. Observe that there exists $c>0$ such that

$$
c^{-1} \cdot \sharp A_{T}\left(g^{\prime}, n, 3 \Delta_{0}\right) \leq \sharp A_{T}\left(g, n, 3 \Delta_{0}\right) \leq c \cdot \sharp A_{T}\left(g^{\prime}, n, 3 \Delta_{0}\right)
$$

for any $g, g^{\prime} \in T$ and $n \geq 0$. Indeed, this is a direct consequence of Lemma 3.7 that $T$ has certain periodicity. Moreover, we claim that

Claim. The following inequality holds

$$
\sharp A_{T}\left(1, n+m, 3 \Delta_{0}\right) \leq c \cdot \sharp A_{T}\left(1, n, 3 \Delta_{0}\right) \cdot \sharp A_{T}\left(1, m, 3 \Delta_{0}\right),
$$

for $n, m \geq 0$. 
Proof of the Claim. For $h \in A_{T}\left(n+m, 3 \Delta_{0}\right)$, we connect 1 and $h$ by a geodesic $[1, h]$ in $\mathcal{T}$. Assume that $d(1, h)=m+n+3 \Delta_{1}$ for some $\left|\Delta_{1}\right| \leq \Delta_{0}$. Let $z \in[1, h]$ such that $d(1, z)=n+3 / 2 \cdot \Delta_{1}$. Note that $z$ might not be in $T$. However, by Lemma 3.7 4$)$, there exists $w \in T$ such that $d(z, w) \leq \Delta+L+2 R=\Delta_{0}$ and then $d(w, h) \leq m+3 \Delta_{0}$. This implies that $w \in A\left(1, n, 3 \Delta_{0}\right)$ and $h \in A_{T}\left(w, m, 3 \Delta_{0}\right)$. The conclusion thus follows from 24].

Define $a_{n}=c \cdot \sharp A_{T}\left(1, n, 3 \Delta_{0}\right)$. The above Claim implies that $a_{n+m} \leq a_{n} a_{m}$. So the sequence $\left(\log a_{n}\right)_{n}$ is subadditive. Then by Fekete Lemma $\lim _{n \rightarrow \infty} \frac{\log a_{n}}{n}=$ $\inf \left\{\frac{\log a_{n}}{n}: n \geq 1\right\}$. Since $\left(a_{n}\right)_{n}$ is non-decreasing we have $a_{n} \leq \sum_{0 \leq i \leq n} a_{i} \leq n a_{n}$. So

$$
\delta_{T, S}=\limsup _{n \rightarrow \infty} \frac{\log \sum_{0 \leq i \leq n} a_{i}}{n}=\lim _{n \rightarrow \infty} \frac{\log a_{n}}{n}=\inf \left\{\frac{\log a_{n}}{n}: n \geq 1\right\} .
$$

It follows that $\sharp A_{T}\left(1, n, \Delta_{0}\right) \geq c^{-1} \exp \left(n \delta_{T, S}\right)$ for $n \geq 1$. Observe that

$$
\Theta_{T}(s, 1) \asymp_{L, \Delta} \sum_{n \geq 0} \sharp A_{T}\left(1, n, \Delta_{0}\right) \cdot \exp (-s n), s \geq 0,
$$

whenever both parts are finite. Thus, $\Theta_{T}(s, 1)$ is divergent at $s=\delta_{T, S}$.

To prove the second statement we estimate the lower bound of $\delta_{T, S}$. By Lemma 3.7. we notice that

$$
\sharp(B(1, i(L+\Delta)) \cap T) \geq \theta^{i} \cdot \exp \left(i \cdot \delta_{G, S} \cdot L\right),
$$

for $i \geq 0$. This implies that

$$
\delta_{T, S} \geq \frac{\log \sharp B_{T}(1, i(L+\Delta)) \cap T}{i(L+\Delta)} \geq \frac{L \cdot \delta_{G, S}+\log \theta}{L+\Delta} .
$$

We obtain $\lim _{L \rightarrow \infty} \delta_{T, S} \geq \delta_{G, S}$. Since $\delta_{T, S} \leq \delta_{G, S}(\forall L)$, the lemma follows.

3.2. Patterson-Sullivan measures on the space of ends of an iterated transitional tree. In this and next subsections, for any $L \gg 0$, let $T$ and $\mathcal{T}$ be the iterated tree set and transitional tree respectively given by Lemma 3.7. At the same time, assume that they satisfy Lemmas 3.8 and 3.9

We denote by the common notation $\partial T$ the limit set of $T$ in either the Bowditch boundary $\Lambda G$ or in the Floyd boundary $\partial_{\lambda} G$. In this subsection, we shall construct a Patterson-Sullivan measure on $\partial T$.

Consider the set $\mathcal{M}(\widetilde{T})$ of finite Borel measures on $\widetilde{T}:=T \cup \partial T$, which is endowed with the weak-convergence topology. Then $\mu_{n} \rightarrow \mu$ for $\mu_{n} \in \mathcal{M}(\widetilde{T})$ if and only if $\liminf _{n \rightarrow \infty} \mu_{n}(U) \geq \mu(U)$ for any open set $U \subset \widetilde{T}$. Note that a set of uniformly bounded measures in $\mathcal{M}(\widetilde{T})$ is relatively compact.

We first construct a family of measures $\left\{\mu_{v}^{s}\right\}_{v \in T} \subset \mathcal{M}(\widetilde{T})$ supported on $T$. Set

$$
\mu_{v}^{s}=\frac{1}{\Theta_{T}(s, 1)} \sum_{g \in T} \exp (-s d(v, g)) \cdot \operatorname{Dirac}(g),
$$

where $s>\delta_{T, S}$ and $v \in T$. By Lemma 3.8, the measures $\left\{\mu_{v}^{s}\right\}_{v \in T}$ are bounded by a uniform constant depending on $L$. 
By Lemma 3.9. for any $v \in T, \Theta_{T}(s, v)$ is divergent at $s=\delta_{T, S}$. Choose $s_{i} \rightarrow \delta_{T, S}$ such that $\mu_{v}^{s_{i}}$ converge in $\mathcal{M}(\widetilde{T})$. The limit measures $\mu_{v}=\lim \mu_{v}^{s_{i}}$ are called Patterson-Sullivan measures at $v$. Clearly, $\left\{\mu_{v}\right\}_{v \in G}$ are absolutely continuous with respect to each other.

In the sequel, we will write PS-measures as shorthand for Patterson-Sullivan measures.

A horofunction co-cycle $B_{\xi}: G \times G \rightarrow \mathbb{R}$ at conical points $\xi \in \Lambda G$ or $\xi \in \partial_{\lambda} G$ was studied in [27]. The precise definition is not relevant here, but we have the following estimation.

Lemma 3.10. [27, Lemma 2.20] For any $L>0$ there exists $C=C(L)>0$ such that the following holds.

Fix $\xi \in \partial T$. For any $x, y \in G$, there is a neighbourhood $V$ of $\xi$ in $\bar{G}_{\lambda}$ or $G \cup \Lambda G$ such that the following property holds:

$$
\left|B_{\xi}(x, y)-B_{z}(x, y)\right|<C, \forall z \in V \cap G,
$$

where $B_{z}(x, y):=d(z, x)-d(z, y)$.

Remarks. (on the proof) The above statement is proved in [27, Lemma 2.20] for a conical point of the Bowditch boundary, where the constant $C$ is universal (not depending on $L)$. In our setting by Lemma 3.7 there exists an $\left(\epsilon, R, L^{\prime}\right)$-transitional ray in the tree $\mathcal{T}$ ending at $\xi$ in $\partial T$. Then by Lemma 2.12 the constant $R$ is uniform for every $\xi \in \partial T$. So the same proof as [27, Lemma 2.20] works to produce a constant $C=C(L)$.

We have to warn the reader that the constant $C>0$ cannot be made uniform for all conical points for the action $G \curvearrowright \partial_{\lambda} G$ on the Floyd boundary as the action is not necessarily geometrically finite (see the discussion after Lemma 2.12).

End of remarks.

With the help of Lemma 3.10 the following can be proven exactly as Théorème 5.4 in 6$]$.

Lemma 3.11. PS-measures $\left\{\mu_{g}\right\}_{g \in T}$ on $\partial T$ satisfy the following property,

$$
\frac{d \mu_{g}}{d \mu_{h}}(\xi) \asymp_{L} \exp \left(-\delta_{T, S} B_{\xi}(g, h)\right),
$$

for $\mu_{h}$-a.e. points $\xi \in \partial T$ and any $g, h \in T$.

3.3. Shadow Lemma. We shall establish a shadow lemma for $\left\{\mu_{g}\right\}_{g \in T}$ on $\partial T$.

Definition 3.12 (Shadow). The shadow $\Pi_{r}(g)$ at $g \in T$ is the set of points $\xi \in \partial T$ such that there exists SOME geodesic $[1, \xi]$ in $\mathcal{T}$ intersecting $B(g, r)$.

Lemma 3.13 (Shadow Lemma). There exists $r_{0}>0$ such that the following holds

$$
\exp \left(-\delta_{T, S} d(1, g)\right) \prec \mu_{1}\left(\Pi_{r}(g)\right) \prec_{r} \exp \left(-\delta_{T, S} d(1, g)\right)
$$

for any $r>r_{0}$ and $g \in T$.

Remark. In [27] the Shadow lemma was proved for the whole group G. The current lemma describes the shadows of the points $g \in T$ in terms of $\delta_{T, S}$.

Proof. By Lemmas 3.10 and 3.11, there exists $C_{1}=C_{1}(L), C_{2}=C_{2}(L)>0$ such that the following holds

$$
C_{1} \exp \left(-\delta_{T, S} d(1, g)\right) \leq \frac{d \mu_{1}}{d \mu_{g}}(\xi) \leq C_{2} \exp \left(-\delta_{T, S} d(1, g)\right)
$$


HAUSDORFF DIMENSION OF BOUNDARIES OF RELATIVELY HYPERBOLIC GROUPS 25

for $\mu_{1}$-a.e. points $\xi \in \partial T$. So in order to estimate $\mu_{1}\left(\Pi_{r}(g)\right)$ we can do it for $\mu_{g}\left(\Pi_{r}(g)\right)$.

Claim. Given any $\epsilon>0$, there is a constant $r_{0}>0$ such that the following holds

$$
\mu_{g}\left(\partial T \backslash \Pi_{r}(g)\right)<\epsilon
$$

for all $g \in T$ and $r>r_{0}$.

Proof of the Claim. Note that $\Pi_{r}(g)$ is a closed set. We consider the convex cone $\mathcal{C}\left(\partial T \backslash \Pi_{r}(g)\right)$ of $\partial T \backslash \Pi_{r}(g)$, which consists of all geodesic rays in $\mathcal{T}$ originating at 1 and terminating at a point in $\partial T \backslash \Pi_{r}(g)$. Let $V$ be the set of vertices of $T$ in $\mathcal{C}\left(\partial T \backslash \Pi_{r}(g)\right)$.

For any $x \in V$, consider the branch point $o$ of $[1, x]$ and $[1, g]$ in $\mathcal{T}$ (defined in the proof of Lemma 3.8). Since $x \notin \mathcal{C}\left(\Pi_{r}(g)\right)$, we have $d(g, o)>r$. By a similar argument to that of Lemma 3.8 , we get

$$
\Theta_{V}(s, g)=\sum_{x \in V} \exp (-s d(x, g)) \asymp \sum_{r \leq k \leq d(1, g)} \exp (-s k) \cdot \Theta_{T}(s, 1) .
$$

So,

$$
\mu_{g}^{s}(V)=\frac{\Theta_{V}(s, g)}{\Theta_{T}(s, 1)} \asymp \sum_{r \leq k \leq d(1, g)} \exp (-s k),
$$

which tends to 0 when $r \rightarrow \infty$ and $s>\delta_{T, S}$.

Thus, the $\mu_{g}^{s}$-measure of the open set $V \cup\left(\partial T \backslash \Pi_{r}(g)\right)$ can be arbitrarily small for $r$ large enough, and so is $\mu_{g}\left(\partial T \backslash \Pi_{r}(g)\right)$. This proves the claim.

By Lemma 3.8, we have that $\left\{\mu_{g}(\partial T)\right\}_{g \in T}$ are lower and upper bounded by a uniform constant depending on $L$. Let $\eta_{1}=1 / 2 \inf \left\{\mu_{g}(\partial T): g \in T\right\}>0$ and $\eta_{2}=\sup \left\{\mu_{g}(\partial T): g \in T\right\}<\infty$. By the above Claim, there is a constant $r_{0}>0$ such that the following holds

$$
\eta_{1}<\mu_{g}\left(\Pi_{r}(g)\right)<\eta_{2}, \forall r>r_{0}
$$

for all $g \in T$. So $(26)$ implies that

$$
\eta_{1} C_{1} \exp \left(-\delta_{T, S} d(1, g)\right) \leq \mu_{1}\left(\Pi_{r}(g)\right) \leq \eta_{2} C_{2} \exp \left(-\delta_{T, S} d(1, g)\right),
$$

for all $g \in G$. The Lemma is proved.

By Shadow Lemma to estimate the PS-measure of balls we need to compare it with that of shadows a in the boundary $\partial T$. Below, we use the symbol $\lfloor s\rfloor$ to denote the integer part of $s \in \mathbb{R}$. Denote by $B_{\rho_{\lambda, 1}}(\xi, t)$ (resp. $\left.B_{\rho_{\lambda, 1}}(\xi, t)\right)$ the ball in $\partial T$ around $\xi \in \partial T$ of radius $t$ with respect to the metric $\rho_{\lambda, 1}\left(\right.$ resp. $\left.\bar{\rho}_{\lambda, 1}\right)$.

Lemma 3.14 (Shadows $\asymp$ Balls). Let $r_{0}$ given by Lemma 3.13. There exists $0<$ $\lambda_{0}<1$ such that for any $\lambda \in\left[\lambda_{0}, 1\right)$ and $L>0$, there exist $r=r(L, \lambda)>r_{0}$ and $C=C(L, \lambda)>0$ with the following property.

For any $\xi \in \partial T$ and $0<t<\lambda$, the following holds

$$
B_{\rho_{\lambda, 1}}\left(\xi, C^{-1} t\right) \subset \Pi_{r}(g) \subset B_{\rho_{\lambda, 1}}(\xi, C t),
$$

and

$$
B_{\bar{\rho}_{\lambda, 1}}\left(\xi, C^{-1} t\right) \subset \Pi_{r}(g) \subset B_{\bar{\rho}_{\lambda, 1}}(\xi, C t),
$$

where $g \in[1, \xi]$ is chosen such that $d(1, g)=\left\lfloor\log _{\lambda} t\right\rfloor$. 
Proof. Let $\lambda_{0}$ be given by Proposition 2.3. For any $\lambda \in\left[\lambda_{0}, 1[\right.$, we consider the family of Floyd metrics $\left\{\rho_{\lambda, v}\right\}_{v \in G}$.

For any $0<t<\lambda$, let $g \in[1, \xi]$ such that $d(1, g)=\left\lfloor\log _{\lambda} t\right\rfloor$. Thus,

$$
\lambda^{d(1, g)+1}<t \leq \lambda^{d(1, g)} .
$$

By construction of $T$ (see Lemma 3.7 5), we know that $[1, \xi]$ is $(\epsilon, R, L)$-transitional. So there exists an $(\epsilon, R)$-transitional point $z$ in $[1, \xi]$ such that $d(z, g) \leq L$. By Lemma 2.10 2, there exists $\kappa=\kappa(\lambda, \epsilon, R)$ such that $\bar{\rho}_{\lambda, z}(1, \xi) \geq \kappa$. By property (2), we have $\rho_{\lambda, g}(1, \xi) \geq \bar{\rho}_{\lambda, g}(1, \xi) \geq \kappa \cdot \lambda^{L}$.

Set $2 C_{1}=\kappa \cdot \lambda^{L}$ and $r=\max \left\{\phi_{\lambda}\left(C_{1}\right), r_{0}\right\}$ where the function $\phi$ is given in Lemma 2.6. Let $\eta \in B_{\rho_{\lambda, 1}}\left(\xi, C_{1} t\right)$. By property (2), it follows that $\rho_{\lambda, g}(\eta, \xi) \leq$ $\lambda^{-d(g, 1)} \rho_{\lambda, 1}(\eta, \xi) \leq C_{1}$. Then $\rho_{\lambda, g}(1, \eta) \geq C_{1}$ and by Lemma 2.6. we have $d(g,[1, \eta]) \leq$ $r$. So $\eta \in \Pi_{r}(g)$. This proves the first inclusions of (28) and (29) for $C=C_{1}$

Let $\eta \in \Pi_{r}(g)$ so that $d(g,[1, \eta]) \leq r$ for some geodesic $[1, \eta]$. Consequently, there exists $w \in[1, \eta[$ such that $d(1, w)=d(1, g)$ and $d(g, w) \leq 2 r$. By Lemma 2.7 any segment of $[1, \xi]$ is a Floyd geodesic with respect to $\rho_{\lambda, 1}$, so $\rho_{\lambda, 1}(\xi, g)=\frac{\lambda^{d(1, g)}}{1-\lambda}$. Let $\alpha$ be a word geodesic between $w$ and $g$. Every edge of $\alpha$ is in the word distance at most $d(1, g)-2 r$ from 1 . So the Floyd length of $\alpha$ is at most $2 r \cdot \lambda^{d(1, g)-2 r}$. We obtain

$$
\begin{aligned}
\bar{\rho}_{\lambda, 1}(\xi, \eta) \leq \rho_{\lambda, 1}(\xi, \eta) & \leq \rho_{\lambda, 1}(g, \xi)+\rho_{\lambda, 1}(w, \eta)+\rho_{\lambda, 1}(g, w) \\
& \leq 2\left(\frac{1}{1-\lambda}+\frac{r}{\lambda^{2 r}}\right) \cdot \lambda^{d(g, 1)}
\end{aligned}
$$

Let $C_{2}=2 \lambda^{-1}\left(\frac{1}{1-\lambda}+\frac{r}{\lambda^{2 r}}\right)$. Then $\bar{\rho}_{\lambda, 1}(\xi, \eta) \leq \rho_{\lambda, 1}(\xi, \eta) \leq C_{2} t$ and so the second inclusions of (28) and (29) follow.

Setting $C=\max \left\{C_{1}, C_{2}\right\}$ we complete the proof of the lemma.

3.4. Proof of Theorem 1.4. We recapitulate the main results of the previous Subsections in the following.

Proposition 3.15. There exists $\lambda_{0}>0$ such that for every $\lambda \in\left[\lambda_{0}, 1\right)$ and $L \gg 0$, there exist an $L$-iterated tree set $T$ and a $P S$-measure $\mu_{1}$ on $\partial T$ satisfying:

$$
\mu_{1}\left(B_{\rho_{\lambda, o}}(\xi, t)\right) \asymp_{\lambda, L} t^{-\delta_{T, S} / \log \lambda},
$$

for any $\xi \in \partial T$ and $0<t<\lambda$.

Proof. The existence of the tree $T$ is proved in Lemma 3.7. Lemmas 3.13 and 3.14 and direct calculations imply that $\partial T$ is Alhfors $Q$-regular for $Q=-\delta_{T, S} / \log \lambda$ (see the definition in the Introduction). Hence, 30 follows.

By Lemma $2.12 \partial T$ consists of uniformly conical points, so Proposition 3.15 implies the first claim of the Theorem. The statement $Q_{i} \rightarrow \delta_{G, S}(i \rightarrow \infty)$ is proved in Lemma 3.9 . Theorem 1.4 is proved.

\section{Proofs of Theorems 1.1 and 1.2}

We consider the Floyd metric on $\partial_{\lambda} G$ and shortcut metric on $\Lambda G$, where the corresponding Theorems 1.1 and 1.2 are proved with the same argument.

The following lemma giving the upper bound for the Hausdorff dimension is due to Marc Bourdon. We notice that it is a general fact which is true for a finitely generated group $G$ without assuming that it is relatively hyperbolic. 
Lemma 4.1 (M. Bourdon, oral communication). For every $\lambda \in(0,1)$, the Hausdorff dimension $\operatorname{Hdim}_{\rho_{\lambda, 1}}$ of $\partial_{\lambda} G$ (respectively $\operatorname{Hdim}_{\bar{\rho}_{\lambda, 1}}$ of $\Lambda G$ ) with respect to the Floyd metric $\rho_{\lambda, 1}$ (respectively to the shortcut metric $\bar{\rho}_{\lambda, 1}$ is upper bounded by $-\delta_{G, S} / \log \lambda$.

Proof. To give an upper bound, it suffices to prove that $\mathcal{H}^{s}\left(\partial_{\lambda} G\right)=0$ for any fixed $s>-\delta_{G, S} / \log \lambda$.

Define $S_{n}=\{g \in G: d(1, g)=n\}$. For any $g \in S_{n}$, define the cone $\Omega_{g}:=\{\xi \in$ $\left.\partial_{\lambda} G, g \in[1, \xi]\right\}$, where $[1, \xi]$ is a geodesic between 1 and $\xi$.

For any $\xi \in \partial_{\lambda} G$, consider a point $x \in[1, \xi] \cap S_{n}$. By Lemma 2.8 the sub-ray $[x, \xi)$ is a $\rho_{\lambda, 1}$-Floyd geodesic. So $\rho_{\lambda, 1}(x, \xi)=\frac{\lambda^{n}}{1-\lambda}$ for any $\xi \in \partial_{\lambda} G$. Thus, $\left\{\Omega_{g}: g \in S_{n}\right\}$ is an $\varepsilon$-covering of $\partial_{\lambda} G$, where $\varepsilon:=\frac{2 \lambda^{n}}{1-\lambda}$.

For any $t \in]-\frac{\delta_{G, S}}{\log \lambda}, s\left[\right.$, we have $-t \log \lambda>\delta_{G, S}$ and so $\sharp S_{n} \prec_{t} \lambda^{-t n}$ for $n \geq 1$. We obtain for all $n \geq 1$ :

$$
\mathcal{H}^{s}\left(\partial_{\lambda} G\right) \leq \sum_{g \in S_{n}} \varepsilon^{s} \prec \lambda^{(s-t) n}
$$

which then tends 0 as $n \rightarrow \infty$. Thus, $\mathcal{H}^{s}\left(\partial_{\lambda} G\right)=0$ for any $s>-\frac{\delta_{G, S}}{\log \lambda}$. The lemma is proved.

So the upper bound on the Hausdorff dimension of $\partial_{\lambda} G$ and $\Lambda G$ in Theorems 1.1. 1.2 is proved. In the remainder of proofs, we aim to establish the lower bound for the Hausdorff dimension.

Taking into account Proposition 3.15 there exists a universal $\lambda_{0}>0$ such that for each $L \gg 0$, there exist an $L$-iterated tree $T$ and a PS-measure $\mu_{1}$ on $\partial T$ such that (30) holds and $\delta_{T, S} \rightarrow \delta_{G, S}$ as $L \rightarrow \infty$.

The following lemma shows that PS-measures constructed in Section 3 are actually the Hausdorff measures on $\partial T$ with respect to the Floyd metric $\rho_{\lambda, 1}$ restricted on $\partial T$.

Lemma 4.2. Let $\mu_{1}$ be a PS-measure on $\partial T$ in $\Lambda G$ or $\partial_{\lambda} G$. Denote $\sigma=-\delta_{T, S} / \log \lambda$. Then we have

$$
\mathcal{H}_{\sigma}(A) \asymp_{L} \mu_{1}(A) \text {. }
$$

for any subset $A \subset \partial T$.

Proof. In the proof, we assume that $\partial T$ is a subset of the Bowditch boundary. The proof for $\partial T \subset \partial_{\lambda} G$ is similar.

Let $\mathcal{B}$ be an $\varepsilon$-covering of $A$ for $\varepsilon>0$. Then $\mu_{1}(A) \leq \sum_{B \in \mathcal{B}} \mu_{1}(B)$. Let $\varepsilon \rightarrow 0$. By Proposition 3.15, we obtain that $\mu_{1}(A) \prec_{L} \mathcal{H}_{\sigma}(A)$.

For the other inequality, we need to make use of the following well-known covering result. Let $B$ be a metric ball of $\operatorname{radius} \operatorname{rad}(B)$ in a proper metric space $X$. Denote by $5 B$ the union of all balls of radius $2 \cdot \operatorname{rad}(B)$ intersecting $B$ so that $\|5 B\| \leq$ $10 \cdot \operatorname{rad}(B)$. Then by [20, Theorem 2.1] for a family of balls $\mathcal{B}$ in $X$ with uniformly bounded radii there exists a sub-family $\mathcal{B}^{\prime} \subset \mathcal{B}$ of pairwise disjoint balls such that the following holds

$$
\bigcup_{B \in \mathcal{B}} B \subset \bigcup_{B \in \mathcal{B}^{\prime}} 5 B .
$$

Note that $\mu_{1}, \mathcal{H}_{\sigma}$ are Radon measures. Then for any $\tau>0$ there exists a compact set $K$ and an open set $U$ such that $K \subset A \subset U$ and $\mathcal{H}_{\sigma}(U \backslash K)<\tau, \mu_{1}(U \backslash K)<\tau$. 
Set $\epsilon_{0}:=\rho_{1}(K, \Lambda G \backslash U)>0$. For any $0<\epsilon<\epsilon_{0}$, let $\mathcal{B}$ be an $\varepsilon$-covering of $K$. By (31) and Proposition 3.15, there exists a sub-family $\mathcal{B}^{\prime}$ of $\mathcal{B}$ such that

$$
H_{\sigma}(K) \leq \sum_{B \in \mathcal{B}^{\prime}}(\|5 B\|)^{\sigma} \leq \sum_{B \in \mathcal{B}^{\prime}}(10 \cdot \operatorname{rad}(B))^{\sigma} \prec_{L} \mu_{1}(U) .
$$

The condition $\tau \rightarrow 0$ yields $H_{\sigma}(A) \prec \mu_{1}(A)$.

Remark. We note that $\mu_{1}$ is unique in the following sense: if $\mu_{1}, \mu_{1}^{\prime}$ are two PSmeasures, then $d \mu_{1} / d \mu_{1}^{\prime}$ is bounded from up and below.

Lemma 4.2 proves that the Hausdorff dimension of $\partial T$ is equal to $\sigma$. Since $\partial T$ is a subset of the set of uniformly conical points in $\partial_{\lambda} G$ and $\Lambda G$, the dimension $\sigma=-\delta_{T, S} / \log \lambda$ of $\partial T$ gives a lower bound of $\operatorname{Hdim}_{\rho_{\lambda, 1}}\left(\partial_{\lambda}^{u c} G\right)$ and $\operatorname{Hdim}_{\bar{\rho}_{\lambda, 1}}\left(\Lambda^{u c} G\right)$.

Letting $L \rightarrow \infty$, we have $\delta_{T, S} \rightarrow \delta_{G, S}$ by Proposition 3.15 . So,

$$
\operatorname{Hdim}_{\rho_{\lambda, 1}}\left(\partial_{\lambda}^{u c} G\right) \geq-\delta_{G, S} / \log \lambda
$$

and

$$
\operatorname{Hdim}_{\rho_{\lambda, 1}}\left(\Lambda^{u c} G\right) \geq-\delta_{G, S} / \log \lambda .
$$

The proofs of Theorems 1.1 and 1.2 are complete.

\section{Tight Paths and Floyd metrics}

In this section, we shall develop a detailed understanding of shortcut geodesics via a class of well-controlled paths called (generalized) tight paths.

5.1. Tight paths. It is well-known that in hyperbolic spaces, a sufficiently "long" local geodesic becomes globally a quasi-geodesic. This property in general fails for the Cayley graph of a relatively hyperbolic group. V. Gerasimov and L. Potyagailo proposed in [14 a notion of tight paths as a generalization of local geodesics to the relative setting. The following definition is a small modification of it.

Definition 5.1. For $c \geq 1, l>0$, a path $\gamma$ is called $(c, l)$-tight path if for any two points $x, y \in \gamma$ with $d(x, y) \leq l$ the subpath $[x, y]_{\gamma}$ is a $c$-quasi-geodesic.

Remark. This definition is a partial case of [14, Definition 6.1] where a local quasigeodesicity is requested outside of the horospheres only and an additional condition is assumed for the horospheres. So if a path is tight in the sense of Definition 5.1 it is also tight in the sense of [14, Definition 6.1] but not necessarily vice versa. In particular we can use all results proven in 14. In addition, the above definition implies that every subpath of a tight path is a tight path itself, which is not always true in the general case. This stability of the tightness for subpaths will be often used below.

We also stress that the above definition does not coincide with the standard notion of local (quasi-)geodesicity when the assumption that the length of a subpath (and not its diameter) is small implies its (quasi-)geodesicity.

In what follows, to reduce cumbersome quantifiers, we continue to use Convention 2.18 without explicit mention on the constants $\epsilon, R$, which depend on the parameter $c>0$ in tight paths.

We recall the following result about tight paths proved in [14]. 
Lemma 5.2. For any $c \geq 1$, there exist $\kappa=\kappa(c), l_{0}=l_{0}(c)>0$ with the following property.

Let $\gamma$ be a $(c, l)$-tight path for $l \geq l_{0}$. Then $\rho_{v}\left(\gamma_{-}, \gamma_{+}\right) \geq \kappa$ for any $(\epsilon, R)$ transitional point $v \in \gamma$.

Comments on the proof. The statement that $\rho_{v}\left(\gamma_{-}, \gamma_{+}\right) \geq \kappa$ is first established in [14. Proposition 6.7] for a special sub-sequence of transitional vertices $v \in \gamma$. Then it is shown in the proof of [14, Theorem B] that the tigthtness of a path implies it for every transitional vertex (up to decreasing the constant $\kappa$ ).

We call below a sequence of points $z_{i}=\gamma\left(t_{i}\right)$ of a length-parametrized path $\gamma$ well-ordered if $t_{i}>t_{i-1}\left(t_{i} \in \mathbb{Z}\right)$.

The following lemma is an intermediate step in the proof of Proposition 5.6 below which is the main result of this subsection.

Lemma 5.3 (Transitional tight path is quasi-geodesic). For any $c, L \geq 0$, there exist $l_{0}=l_{0}(L), c^{\prime}=c^{\prime}(c) \geq 1$ with the following property.

Let $\gamma$ be a $(c, l)$-tight path for $l \geq l_{0}$. Assume that $\gamma$ is an $(\epsilon, R, L)$-transitional path, where $\epsilon, R$ satisfy Convention 2.18. Then $\gamma$ is a $c^{\prime}$-quasi-geodesic.

Proof. By Lemma 5.2 , there exists $\kappa=\kappa(c) \geq 0$ such that $\rho_{x}\left(\gamma_{-}, \gamma_{+}\right) \geq \kappa$ for every $(\epsilon, R)$-transitional point $x \in \gamma$. Set $D_{0}=\phi(\kappa / 2)$. Choose $l_{0} \geq 2\left(L+D_{0}\right)$.

Since any subpath of $\gamma$ is $(c, l)$-tight, it is enough to prove that there exists a linear bound for $\ell(\gamma)$ with respect to $d\left(\gamma_{-}, \gamma_{+}\right)$. Let $\alpha$ be a geodesic with the same endpoints as $\gamma$. The idea of proof is to find two sequences of well-ordered points in $\gamma$ and $\alpha$ respectively which are uniformly close.

Since $\gamma$ is $(\epsilon, R, L)$-transitional, there exists a maximal set of $(\epsilon, R)$-transitional well-ordered points $\left\{z_{i}: 1 \leq i \leq n\right\}$ in $\gamma$ such that

$$
\ell\left(\left[z_{i}, z_{j}\right]_{\gamma}\right) \geq 2 D_{0}
$$

for $i \neq j$ and

$$
\ell\left(\left[z_{i}, z_{i+1}\right]_{\gamma}\right) \leq 2\left(L+D_{0}\right)
$$

for $1 \leq i<n$. Indeed, let $z_{1}$ be the first $(\epsilon, R)$-transitional point in $\gamma$. Suppose $z_{i}$ is chosen for $i \geq 1$. If $\ell\left(\left[z_{i}, \gamma_{+}\right]\right) \leq 2\left(L+D_{0}\right)$ then $z_{i+1}=\gamma_{+}$. Consider the point $z$ in $\left[z_{i}, \gamma_{+}\right]_{\gamma}$ such that $\ell\left(\left[z_{i}, z\right]_{\gamma}\right)=L+2 D_{0}$. If $z$ is $(\epsilon, R)$-transitional in $\gamma$, then set $z_{i+1}=z$. Otherwise, there exists an $(\epsilon, R)$-transitional point $z_{i+1}$ such that $\ell\left(\left[z, z_{i+1}\right]_{\gamma}\right) \leq L$ and $\ell\left(\left[z_{i}, z_{i+1}\right]_{\gamma}\right) \leq 2\left(L+D_{0}\right)$.

By Lemma 5.2 , there exists $\kappa>0$ such that

$$
\rho_{z_{i+1}}\left(z_{i}, \gamma_{+}\right) \geq \kappa
$$

for any $1 \leq i<n$. By Lemma 2.6 there exists $w_{1} \in \alpha$ such that $d\left(z_{1}, w_{1}\right) \leq D_{0}$. We now choose other $w_{i}$ inductively for $i \geq 1$.

Suppose $w_{i} \in \alpha$ is chosen such that $d\left(z_{i}, w_{i}\right) \leq D_{0}$. Since $d\left(z_{i+1}, z_{i}\right) \geq 2 D_{0}$, we obtain $\left[z_{i}, w_{i}\right] \cap B\left(z_{i+1}, D_{0}\right)=\emptyset$. By the choice of $D_{0}=\phi(\kappa / 2)$, we know that for any $v \in G$, any geodesic outside $B\left(v, D_{0}\right)$ has $\mathfrak{l}_{v}$-length at most $\kappa / 2$. So $\rho_{z_{i+1}}\left(w_{i}, z_{i}\right) \leq \kappa / 2$ and then $\rho_{z_{i+1}}\left(w_{i}, \gamma_{+}\right) \geq \kappa / 2$. Thus there exists $w_{i+1} \in$ $\left[w_{i}, \alpha_{+}\right]_{\alpha}$ such that $d\left(z_{i+1}, w_{i+1}\right) \leq D_{0}$. Clearly, the obtained points $w_{i}$ are wellordered on $\alpha$.

As $l_{0}>2\left(L+D_{0}\right),\left[z_{i}, z_{i+1}\right]_{\gamma}$ is a $c$-quasi-geodesic by the tightness property. Since $w_{i}$ are well-ordered on $\alpha$, we see that $\gamma$ is a $c^{\prime}$-quasi-geodesic for $c^{\prime}:=c+2 D_{0}$.

The following lemma will be often used further. 
Lemma 5.4 (Bounded overlap). For $c \geq 1$ and $(\epsilon, R)$ given by Convention 2.18, there exist $K_{0}, l_{0}>0$ with the following property.

Let $\gamma$ be a $(c, l)$-tight path for $l \geq l_{0}$. Assume that $\beta_{1}, \beta_{2}$ are two maximal connected segments of $\gamma$ such that $\left(\beta_{i}\right)_{-},\left(\beta_{i}\right)_{+} \in N_{\epsilon}\left(X_{i}\right)$ for some $X_{i} \in \mathbb{P}$ with $i=1,2$. Then $\ell\left(\beta_{1} \cap \beta_{2}\right) \leq K_{0}$. In particular, the endpoints of $\beta_{i}$ are $(\epsilon, R)$ transitional for $i=1,2$.

Proof. By Definition 5.1 a subpath of a tight path is itself tight. Then by [14, Proposition 7.6] it follows that there exists $l_{0}>0$ such that for all $l \geq l_{0}$ the elements of $\mathbb{P}$ are uniformly quasi-convex with respect to the system of $(c, l)$-tight paths. This implies that there exists a uniform constant $\varepsilon=\varepsilon(\epsilon, c)>0$ such that $\beta_{i} \subset N_{\varepsilon}\left(X_{i}\right)$ for $i=1,2$. By Lemma 2.16 we find a constant $R=\mathcal{R}(\varepsilon)>0$ such that $\left\|N_{\varepsilon}(X) \cap N_{\varepsilon}\left(X^{\prime}\right)\right\| \leq R$ for every $X, X^{\prime} \in \mathbb{P}$. Assume that $l_{0}>c R+c$. Since $\beta_{i}$ are $l$-local $c$-quasi-geodesic for $l>l_{0}$, it follows that $\ell\left(\beta_{1} \cap \beta_{2}\right) \leq K_{0}:=c R+c$.

Remark. By the bounded intersection of $\mathbb{P}$, this lemma holds trivially if $\gamma$ is a quasi-geodesic. However, the tight path $\gamma$ above is a local quasi-geodesic only.

Let $\gamma$ be a $(c, l)$-tight path. Let $\epsilon=\epsilon(c)$ given by Convention 2.18 and $K_{0}$ given by Lemma 5.4. For $K>K_{0}$, we consider all maximal connected segments $\beta_{i}$ in $\gamma(1 \leq i \leq m)$ such that $\ell\left(\beta_{i}\right) \geq K$ and $\left(\beta_{i}\right)_{-},\left(\beta_{i}\right)_{+} \in N_{\epsilon}\left(X_{i}\right)$ for some $X_{i} \in \mathbb{P}$. Consequently, $X_{i} \neq X_{j}$ for $i \neq j$. These $\left(\beta_{i}, X_{i}\right)$ shall be referred to as $(\epsilon, K)$-components of $\gamma$.

We stress that by the argument of Lemma 5.4 the segment $\beta_{i}$ belongs to $N_{\epsilon}\left(X_{i}\right)$ for a uniform $\epsilon>0$ and unique $X_{i}$.

We now introduce a modification of a tight path to make the obtained path a quasi-geodesic.

Definition 5.5 (Truncation of a tight path). Let $\gamma$ be a $(c, l)$-tight path for $c \geq$ $1, l>0$. Consider all $(\epsilon, K)$-components $\left(\beta_{i}, X_{i}\right)(1 \leq i \leq m)$ for a fixed $K>2 K_{0}$, where $K_{0}>0$ is given by Lemma 5.4 .

Set $y_{1}=\left(\beta_{1}\right)_{-}, x_{2}=\left(\beta_{1}\right)_{+}$. If $\beta_{i} \cap \beta_{i-1}=\emptyset$ for $i \geq 2$, denote $y_{i}=\left(\beta_{i}\right)_{-}, x_{i+1}=$ $\left(\beta_{i}\right)_{+}$; otherwise, set $y_{i}=x_{i-1}, x_{i+1}=\left(\beta_{i}\right)_{+}$. Replace $\left[y_{i}, x_{i+1}\right]_{\gamma}$ by a geodesic segment $\left[y_{i}, x_{i+1}\right]$ for each $i \geq 1$.

The path $\bar{\gamma}$ obtained in this way is called a $K$-truncation of $\gamma$.

Remark. The following observation is elementary and useful: every $\beta_{i}$ produces an $(\epsilon, K / 2)$-deep point in $X_{i}$ in the truncation path $\bar{\gamma}$. Consequently, if $\bar{\gamma}$ does not contain an $(\epsilon, R)$-deep point, then $d\left(\left(\beta_{i}\right)_{-},\left(\beta_{i}\right)_{+}\right) \leq 2 R$ for all $\beta_{i}$.

The following lemma is the main result of this subsection. It provides a further generalization of Lemma 5.3 to the truncated tight paths.

Lemma 5.6 (Truncation is quasi-geodesic). For any $c \geq 1$, there exist $l_{0}=l_{0}(c), K=$ $K(c), c^{\prime}=c^{\prime}(c)>0$ with the following property. For any $l \geq l_{0}$, the $K$-truncation of a $(c, l)$-tight path is a $c^{\prime}$-quasi-geodesic.

Proof. Let $K>2 K_{0}$ be a fixed integer, where $K_{0}$ is given by Lemma 5.4. Let $\bar{\gamma}$ be the $K$-truncation of a $(c, l)$-tight path $\gamma$. Keeping the notations as in Definition 5.5 , we have by Lemma 5.4 that $y_{i}, x_{i+1}$ for $1 \leq i \leq m$ are $(\epsilon, R)$-transitional points in $\gamma$. Furthermore since $\left[x_{i}, y_{i}\right]_{\gamma}$ contains no $(\epsilon, K)$-components for $1 \leq i<m$, we see that $\left[x_{i}, y_{i}\right]_{\gamma}$ is an $(\epsilon, R, L)$-transitional path for $L:=K / 2$. By Lemma 5.3, there exist $l_{0}=l_{0}(L), c_{0}=c_{0}(c) \geq 1$ such that $\left[x_{i}, y_{i}\right]_{\gamma}$ is a $c_{0}$-quasi-geodesic. 
The geodesic $\left[y_{i}, x_{i+1}\right]$ belongs to the $\epsilon$-neighbourhood $N_{\epsilon}\left(X_{i}\right)$ for some $X_{i} \in \mathbb{P}$ where we assume that $y_{i}$ is the entry point of $\gamma \cap N_{\epsilon}(X)$ and $x_{i+1}$ is the exit point of it. We will now show that $\gamma$ is a quasi-geodesic in a neighbourhood of $y_{i}$.

Since $y_{i}$ is $(\epsilon, R)$-transitional, $\rho_{y_{i}}\left(x_{i}, x_{i+1}\right) \geq \kappa$ where $\kappa$ is given by Lemma 5.2 . Hence, $d\left(y_{i},\left[x_{i}, x_{i+1}\right]\right) \leq D_{0}:=\phi(\kappa)$ where $\phi$ is the function given by Lemma 2.6. Connect the point $x_{i}$ with an arbitrary point $z \in\left[y_{i}, x_{i+1}\right]$ by a geodesic $\beta$. By the triangle inequality we have $d\left(x_{i}, y_{i}\right)+d\left(y_{i}, x_{i+1}\right) \leq d\left(x_{i}, x_{i+1}\right)+2 D_{0}$. Since $d\left(y_{i}, z\right)+d\left(z, x_{i+1}\right)=d\left(y_{i}, x_{i+1}\right)$ we obtain $d\left(x_{i}, y_{i}\right)+d\left(y_{i}, z\right) \leq d\left(x_{i}, x_{i+1}\right)-$ $d\left(z, x_{i+1}\right)+2 D_{0} \leq d\left(x_{i}, z\right)+2 D_{0}$. Finally

$$
\begin{aligned}
\ell\left(\left[x_{i}, z\right]_{\bar{\gamma}}\right) & =\ell\left(\left[x_{i}, y_{i}\right]_{\gamma}\right)+\ell\left(\left[y_{i}, z\right]\right) \\
& \leq c_{0} d\left(x_{i}, y_{i}\right)+c_{0}+d\left(y_{i}, z\right) \\
& \leq c_{0} d\left(x_{i}, z\right)+c_{0}+2 c_{0} D_{0} .
\end{aligned}
$$

So $\left[x_{i}, z\right]_{\bar{\gamma}}$ is a $c_{1}$-quasi-geodesic for $c_{1}:=c_{0}\left(2 D_{0}+1\right)$.

We have that any subpath $\tilde{\gamma}$ of the truncated path $\bar{\gamma}$ is the union of three types of $c_{1}$-quasi-geodesic subpaths: a) $\gamma_{i}=\left[x_{i}, y_{i}\right]_{\gamma}$, b) $\beta_{i}=\left[y_{i}, x_{i+1}\right]_{\gamma}$ and c) $\delta=[a, b]$. Both vertices of the intervals of types a) and b) are transitional on the corresponding tight path $\gamma$, and every $\gamma_{i}$ is $(\epsilon, R, L)$-transitional whereas $\beta_{i}$ is an $(\epsilon, K)$-component. The path $\tilde{\gamma}$ can contain at most two intervals $\delta$ of type $c$ ) such that one of the endpoints of $\delta$ coincides with an endpoint of $\tilde{\gamma}$ and is an interior point of a geodesic truncation of $\bar{\gamma}$.

Repeating the argument of Lemma 5.3 consider a maximal well-ordered subset $V$ of the transitional vertices $\left\{v_{j} \in \tilde{\gamma}\right\}$ in the set $W:=\tilde{\gamma} \cap\left\{y_{i}, x_{i+1}: 1 \leq i \leq m\right\}$ such that $d\left(v_{j}, v_{j+1}\right) \geq 2 D_{0}$. We connect the endpoints of $\tilde{\gamma}$ by a geodesic $\alpha$. Then for each $v_{j} \in V$, there exists $v_{j}^{\prime} \in \alpha$ such that $d\left(v_{j}, v_{j}^{\prime}\right) \leq D_{0}$ and $v_{j}^{\prime} \in\left[v_{j-1}^{\prime}, v_{j+1}^{\prime}\right]_{\alpha}$. Since $V$ is maximal in $W$, for any $w \in W$ there exists $v \in V$ such that $d(v, w) \leq 2 D_{0}$. If $W=\left\{w_{1}, w_{2}, \cdots, w_{n}\right\}$, there exists a well-ordered set $W^{\prime}=\left\{w_{1}^{\prime}, w_{2}^{\prime}, \cdots, w_{n}^{\prime}\right\}$ of vertices in $\alpha$ such that $d\left(w_{i}, w_{i}^{\prime}\right) \leq 3 D_{0}$. Then $\left[\tilde{\gamma}_{-}, w_{1}\right]_{\tilde{\gamma}},\left[w_{i}, w_{i+1}\right]_{\gamma}$ and $\left[w_{n}, \tilde{\gamma}_{+}\right]_{\tilde{\gamma}}$ are all $c_{1}$-quasi-geodesics by the above argument. We have

$$
\begin{aligned}
\ell(\tilde{\gamma}) & \leq c_{1}\left(d\left(\tilde{\gamma}_{-}, w_{1}\right)+\sum_{i=1}^{n-1} d\left(w_{i}, w_{i+1}\right)+d\left(w_{n}, \tilde{\gamma}_{+}\right)\right)+c_{1} \\
& \leq c_{1}\left(3 D_{0}+d\left(\tilde{\gamma}_{-}, w_{1}^{\prime}\right)+\sum_{i=1}^{n-1}\left(d\left(w_{i}^{\prime}, w_{i+1}^{\prime}\right)+3 D_{0}\right)+d\left(w_{n}^{\prime}, \tilde{\gamma}_{+}\right)+3 D_{0}\right)+c_{1} \\
& \leq c^{\prime} d\left(\tilde{\gamma}_{-}, \tilde{\gamma}_{+}\right)+c^{\prime}
\end{aligned}
$$

where $c^{\prime}:=\left(1+3 D_{0}\right) c_{1}$. The Lemma is proved.

Convention 5.7. For any $c \geq 1$, we will assume further on that $l_{0}, K>0$ satisfy both Lemmas 5.2 and 5.4

5.2. Shortcut metrics and generalized tight paths. Recall that a Floyd geodesic in the Floyd completion does not in general belongs to the Cayley graph and the shortening procedure described in subsection 2.3 allows one to approximate them by local geodesics in the graph. Furthermore the following lemma shows that this approximation can be done using the tight paths:

Lemma 5.8. [14, Corollary 7.8] For any $l>0$ there exists $\left.\lambda_{0} \in\right] 0,1[$ such that for every $\lambda \in] \lambda_{0}, 1\left[\right.$ if the Floyd geodesic $\gamma \subset \bar{G}_{\lambda}$ (with respect to the metric $\rho_{\lambda, o}$ ) joining two distinct points $x, y$ in $\bar{G}_{\lambda}$ does not belong to the Cayley graph $\mathscr{G}(G, S)$, then for $\varepsilon>0$ there exists a tight path $\tilde{\gamma} \subset \mathscr{G}(G, S)$ such that $\left|\mathfrak{l}_{\lambda, o}(\tilde{\gamma})-\mathfrak{l}_{\lambda, o}(\gamma)\right|<\varepsilon$. 
The goal of this subsection is to extend this result to the geodesics with respect to the shortcut metrics $\left\{\bar{\rho}_{\lambda, o}\right\}_{o \in G}$ on $\Lambda G$ (see Section 2.2). For this purpose we generalize the notion of a tight path as follows.

Definition 5.9 (Generalized tight paths and truncations). Let $\gamma$ be a finite sequence of $(c, l)$-tight paths $\gamma_{i}$ in $\mathscr{G}(G, S)(1 \leq i \leq n)$ such that $\left(\gamma_{i}\right)_{+},\left(\gamma_{i+1}\right)_{-} \in N_{\epsilon}\left(X_{i}\right)$ for some $X_{i} \in \mathbb{P}$ where $X_{i} \neq X_{j}(1 \leq i \neq j<n)$.

We say that $\gamma$ is a $(c, l)$-generalized tight path if for each pair of entry and exit points $y_{i}, x_{i+1}$ of $\gamma_{i}$ and $\gamma_{i+1}$ respectively in $N_{\epsilon}\left(X_{i}\right)$ we have $d\left(y_{i}, x_{i+1}\right) \geq l$ $(1 \leq i<n)$.

Fix $K>0$. For $n>1$, consider the $K$-truncation $\bar{\gamma}_{i}$ of $\left[x_{i}, y_{i}\right]_{\gamma_{i}}$ where $1 \leq i \leq n$. The path

$$
\tilde{\gamma}=\bar{\gamma}_{1} \cdot\left[y_{1}, x_{2}\right] \cdot \bar{\gamma}_{2} \cdots\left[y_{n-1}, x_{n}\right] \cdot \bar{\gamma}_{n}
$$

is called the $K$-truncation of a generalized $(c, l)$-tight $\gamma$.

Remark. Note that a generalized tight path is possibly not connected. If it is connected, then it is a tight path in Definition 5.1 .

Lemma 5.10 (Generalized truncation is quasi-geodesic). For any $c \geq 1$, there exist $l_{0}, K, c^{\prime} \geq 1$ such that for any $l>l_{0}$, the $K$-truncation of a $(c, l)$-generalized tight path is a $c^{\prime}$-quasi-geodesic.

Proof. Let $K=K(c)$ be given by Lemma 5.6 . Let $\tilde{\gamma}$ be the $K$-truncation of a generalized $(c, l)$-tight path $\gamma$. We keep the notations of Definition 5.9. If $n=1$, the proof is finished by Lemma 5.6 . Assume that $n \geq 2$.

By Lemma 5.6, there exists $c_{1}>0$ such that each $\bar{\gamma}_{i}$ is a $c_{1}$-quasi-geodesic for each $1 \leq i<n$. We prove below that $\bar{\gamma}_{i}$ and $\bar{\gamma}_{i+1}$ have bounded projection to $N_{\epsilon}\left(X_{i}\right)$ where $X_{i} \in \mathbb{P}$.

By Lemma 2.16, $X \in \mathbb{P}$ is $c_{1}$-contracting and there exist $\mu_{c_{1}}, D_{c_{1}}>0$ such that the (7) holds. By Convention 2.18, we have $\epsilon \geq \mu_{c_{1}}$. Let $z$ be the entry point of $\bar{\gamma}_{i}$ in $N_{\epsilon}\left(X_{i}\right)$.

Claim. There exists a constant $C>0$ such that $d\left(z, y_{i}\right) \leq C$.

Proof of Claim. Since $y_{i}$ is the entry point of $\gamma_{i}$ in $N_{\epsilon}\left(X_{i}\right)$, there exists an $(\epsilon, K)$ component $\beta$ of $\gamma_{i}$ such that $z \in \beta$. Consider the tight subpath $\left[\beta_{+}, y_{i}\right]_{\gamma_{i}}$ and its $K$-truncation $\beta_{1}$. By the argument in Lemma 5.6 the path $\left[z, \beta_{+}\right] \cdot \beta_{1}$ is a $c_{2}$-quasi-geodesic for some $c_{2}>0$.

Since $X_{i}$ is quasi-convex, there exists $\varepsilon=\varepsilon\left(\epsilon, c_{2}\right)>0$ such that any $c_{2}$-quasigeodesic with two endpoints in $N_{\epsilon}\left(X_{i}\right)$ lies in $N_{\varepsilon}\left(X_{i}\right)$. This implies that $\beta_{1} \subset$ $N_{\varepsilon}\left(X_{i}\right)$. However, there exists no $(\epsilon, K)$-components in $\left[\beta_{+}, y_{i}\right]_{\gamma_{i}}$. Indeed, if not, there exists an $(\epsilon, K)$-component $\beta^{\prime}$ in $\left[\beta_{+}, y_{i}\right]_{\gamma_{i}}$ and $Y \in \mathbb{P}$ such that $\beta_{ \pm}^{\prime} \in N_{\epsilon}(Y)$ and $d\left(\beta_{-}^{\prime}, \beta_{+}^{\prime}\right)>K>l_{0}$. Since $y_{i}$ is the entry point of $\gamma_{i}$ in $N_{\epsilon}\left(X_{i}\right)$, we have $Y \neq X_{i}$. Since $\beta_{ \pm}^{\prime} \in N_{\varepsilon}\left(X_{i}\right)$, we get $d\left(\beta_{-}^{\prime}, \beta_{+}^{\prime}\right) \leq R:=\mathcal{R}(\max \{\epsilon, \varepsilon\})$ by Lemma 2.16. This is a contradiction as $l_{0}>\mathcal{R}(\max \{\epsilon, \varepsilon\})$. The same reasoning shows that $d\left(z, \beta_{+}\right) \leq R$.

Let $L=L(\varepsilon, 1)$ be given by Lemma 2.17. If $d\left(z, y_{i}\right)>2 L+R$, there exists an interior point in $\left[\beta_{+}, y_{i}\right]_{\gamma_{i}}$ which is $\left(\epsilon_{c}, 1\right)$-deep in $X_{i}$. This is a contradiction, since $y_{i}$ is the entry point of $\left[\beta_{+}, y_{i}\right]_{\gamma_{i}}$ in $N_{\epsilon}\left(X_{i}\right)$ and $\epsilon \geq \epsilon_{c}$ by Convention 2.18. Hence we proved that $d\left(z, y_{i}\right) \leq C:=2 L+R$. The claim is proved.

By the contracting property 2.16 we see that $\operatorname{Proj}_{X_{i}}\left(\bar{\gamma}_{i}\right) \leq \tau:=2\left(D_{c_{1}}+\epsilon\right)+C$. The same is true for $\mathbf{P r o j}_{X_{i}}\left(\bar{\gamma}_{i+1}\right)$. Then $\tilde{\gamma}$ satisfies the following properties: 
(1) Each $\bar{\gamma}_{i}$ is a $c_{1}$-quasi-geodesic,

(2) $\max \left\{\operatorname{Proj}_{X_{i}}\left(\bar{\gamma}_{i}\right), \operatorname{Proj}_{X_{i}}\left(\bar{\gamma}_{i+1}\right)\right\} \leq \tau$,

(3) $d\left(x_{i}, y_{i+1}\right)>l$.

(These properties imply that $\tilde{\gamma}$ is $\left(l, c_{1}, c_{1}, \tau\right)$-admissible in the sense of [28, Section $3])$. Therefore by Corollary 3.3 in [28, there exist $l_{0}, c^{\prime}>0$ such that for any $l>l_{0}$ the truncation $\tilde{\gamma}$ is a $c^{\prime}$-quasi-geodesic. The lemma is proved.

Remark. An alternative way to prove the above Lemma is to use the arguments of Proposition 6.1.1 in [15] to prove that $\tilde{\gamma}$ is a curve whose distortion is a quadratic polynomial, then it follows from Proposition 7.2.2 in [15] that $\tilde{\gamma}$ is linearly distorted.

Proposition 5.11 (Approximation by generalized tight paths). For any $l \geq 0$, there exists $0<\lambda_{0}<1$ such that the following property holds for any $\lambda \in\left[\lambda_{0}, 1\right)$.

For any $\xi \neq \eta \in \Lambda G$, there exists a sequence of generalized $(1, l)$-tight paths $\gamma_{n}$ with $\left(\gamma_{n}\right)_{-} \in[o, \xi],\left(\gamma_{n}\right)_{+} \in[o, \eta]$ such that

$$
\lim _{n \rightarrow \infty} d\left(o,\left(\gamma_{n}\right)_{-}\right)=\lim _{n \rightarrow \infty} d\left(o,\left(\gamma_{n}\right)_{+}\right)=\infty
$$

and

$$
\lim _{n \rightarrow \infty} \mathfrak{l}_{\lambda, o}\left(\gamma_{n}\right)=\bar{\rho}_{\lambda, o}(\xi, \eta) .
$$

Proof. By definition of the shortcut metric (3), for any $\varepsilon>0$, there are finitely many pairs $\left(\eta_{i}, \xi_{i+1}\right) \in \omega$ where $1 \leq i<m$ such that

$$
\bar{\rho}_{\lambda, o}(\xi, \eta) \geq \sum_{1 \leq i \leq m} \rho_{\lambda, o}\left(\xi_{i}, \eta_{i}\right)-\varepsilon / 3
$$

where $\xi_{1}:=\xi, \eta_{m}:=\eta$. If $m=1$, the proof is completed by Lemma 5.8, Assume that $m \geq 2$.

Let $\kappa=\min \left\{\rho_{o}\left(\eta_{i}, \xi_{i+1}\right): 1 \leq i<m\right\}>0$. For each $1 \leq i<m$, there exists $X_{i} \in \mathbb{P}$ such that $\eta_{i}, \xi_{i+1} \in \partial_{\lambda}\left(X_{i}\right)$ where $\partial_{\lambda}\left(X_{i}\right)$ is the topological boundary of $X_{i}$ in $\partial_{\lambda} G$.

First we claim that one can choose $\tilde{\xi}_{1}, \tilde{\eta}_{m}$ and $\tilde{\eta}_{i}, \tilde{\xi}_{i+1} \in X_{i}$ for each $1 \leq i<m$ such that the following two conditions hold,

(1) $\max \left\{\rho_{o}\left(\tilde{\xi}_{i}, \xi_{i}\right), \rho_{o}\left(\tilde{\eta}_{i}, \eta_{i}\right)\right\} \leq \min \left\{\kappa / 4, \frac{\varepsilon}{6 m}\right\}$ for $1 \leq i \leq m$.

(2) If there exists a path $\alpha$ between $\tilde{\eta}_{i}, \tilde{\xi}_{i+1}$ for $1 \leq i<m$ such that $\ell(\alpha) \leq 3 l$, then it has $\mathfrak{l}_{o}$-length at most $\kappa / 4$.

Indeed, (1) is true for $\tilde{\xi}_{i}$ and $\tilde{\eta}_{i}$ sufficiently close to $\xi_{i}$ and $\eta_{i}$ respectively. To prove (2), let $R=\min \left\{d\left(1, \tilde{\xi}_{i}\right), d\left(1, \tilde{\eta}_{i}\right): 1 \leq i \leq m\right\}$. We have $d(1, \alpha) \geq R-3 l$. So for sufficiently large $R$ the statement (2) follows from the visibility lemma 2.6 .

By Lemma 5.8, we can connect $\tilde{\xi}_{i}, \tilde{\eta}_{i}$ by a $(1, l)$-tight path $\gamma_{i}$ for $1 \leq i \leq m$ such that $\left(\gamma_{i}\right)_{-}=\xi_{i}$ and $\left(\gamma_{i}\right)_{+}=\tilde{\eta}_{i}$ and

$$
\left|\rho_{\lambda, o}\left(\tilde{\xi}_{i}, \tilde{\eta}_{i}\right)-\mathfrak{l}_{\lambda, o}\left(\gamma_{i}\right)\right| \leq \frac{\varepsilon}{6 m} .
$$

By the condition (1) above, 32 and (33) , the following holds

$$
\bar{\rho}_{\lambda, o}(\xi, \eta) \geq \sum_{1 \leq i \leq m} \mathfrak{l}_{\lambda, o}\left(\gamma_{i}\right)-\frac{5 \varepsilon}{6} .
$$

Let $y_{i}, x_{i+1}$ be the entry and exit points of $\gamma_{i}$ and $\gamma_{i+1}$ in $N_{\epsilon}\left(X_{i}\right)$ respectively. If $d\left(y_{i}, x_{i+1}\right) \geq l$ for all $1 \leq i<m$, then we are done: $\left\{\gamma_{i}\right\}$ give the generalized tight path. Otherwise, assume that $d\left(x_{j+1}, y_{j}\right) \leq l$ for some $1 \leq j<m$. 
Observe that $\max \left\{d\left(\tilde{\eta}_{j}, y_{j}\right), d\left(\tilde{\xi}_{j+1}, x_{j+1}\right)\right\} \geq l+1$. Indeed, if not, it follows that $\tilde{\eta}_{j}, \tilde{\xi}_{j+1}$ are connected by a path of length at most $3 l$. By the above condition $(2)$, we have $\rho_{o}\left(\tilde{\eta}_{j}, \tilde{\xi}_{j+1}\right) \leq \kappa / 4$. By the condition $(1)$, we have $\rho_{o}\left(\eta_{j}, \xi_{j+1}\right) \leq$ $3 \kappa / 4$. We arrive at a contradiction with the definition of $\kappa$. Thus, we proved that $\max \left\{d\left(\tilde{\eta}_{j}, y_{j}\right), d\left(\tilde{\xi}_{j+1}, x_{j+1}\right)\right\} \geq l+1$. By Lemma 2.7, we obtain the following

$$
\mathfrak{l}_{\lambda, o}\left(\left[y_{j}, \tilde{\eta}_{j}\right]_{\gamma_{j}}\right)+\mathfrak{l}_{\lambda, o}\left(\left[\tilde{\xi}_{j+1}, x_{j+1}\right]_{\gamma_{j+1}}\right) \geq \mathfrak{l}_{\lambda, o}\left(\left[y_{j}, x_{j+1}\right]\right) .
$$

which yields

$$
\begin{aligned}
\mathfrak{l}_{\lambda, o}\left(\gamma_{j}\right)+\mathfrak{l}_{\lambda, o}\left(\gamma_{j+1}\right) & \geq \mathfrak{l}_{\lambda, o}\left(\left[\tilde{\xi}_{j}, y_{j}\right]_{\gamma_{j}}\right)+\mathfrak{l}_{\lambda, o}\left(\left[y_{j}, x_{j+1}\right]\right)+\mathfrak{l}_{\lambda, o}\left(\left[x_{j+1}, \tilde{\eta}_{j+1}\right]_{\gamma_{j+1}}\right) \\
& \geq \rho_{\lambda, o}\left(\tilde{\xi}_{j}, \tilde{\eta}_{j+1}\right) .
\end{aligned}
$$

This implies that we can drop the pair $\left(\eta_{j}, \xi_{j+1}\right)$ in 32 such that the corresponding inequality in (34) still holds. Precisely, choose a $(1, l)$-tight path $\alpha_{j}$ between $\tilde{\xi}_{j}, \tilde{\eta}_{j+1}$ such that

$$
\left|\mathfrak{l}_{\lambda, o}\left(\alpha_{j}\right)-\rho_{\lambda, o}\left(\tilde{\xi}_{j}, \tilde{\eta}_{j+1}\right)\right| \leq \frac{\varepsilon}{6 m} .
$$

So $\mathfrak{l}_{\lambda, o}\left(\gamma_{j}\right)+\mathfrak{l}_{\lambda, o}\left(\gamma_{j+1}\right) \geq \mathfrak{l}_{\lambda, o}\left(\alpha_{j}\right)-\frac{\varepsilon}{6 m}$. It follows by 34 ,

$$
\bar{\rho}_{\lambda, o}(\xi, \eta) \geq \sum_{1 \leq i \leq m ; i \neq j, j+1} \mathfrak{l}_{\lambda, o}\left(\gamma_{i}\right)+\mathfrak{l}_{\lambda, o}\left(\alpha_{j}\right)-\frac{5 \varepsilon}{6}-\frac{\varepsilon}{6 m} .
$$

Consider the new set of $(1, c)$-tight paths $\gamma_{i}(i \neq j, j+1)$ and $\alpha_{j}$. Repeat the above argument for those $j$ for which $d\left(x_{j+1}, y_{j}\right) \leq l$. Since $m$ is finite, for every $\varepsilon>0$ we obtain a generalized tight path $\gamma$ such that $\bar{\rho}_{\lambda, o}(\xi, \eta) \geq \mathfrak{l}_{\lambda, o}(\gamma)-\epsilon$. The Proposition is proved.

5.3. Floyd and shortcut metrics on uniformly conical points. A priori, the shortcut metrics as quotient of the Floyd metrics might be distorted in a unexpected way. The main result of this subsection is to show that this distortion is not severe for uniformly conical points.

Fix a basepoint $o \in G$. Recall that, in Section 2.4, $\Lambda_{L, o}^{u c} G$ denotes the set of uniformly conical points $\xi \in \Lambda G$ for which there exists an $(\epsilon, R, L)$-transitional geodesic ray between $o$ and $\xi$. Similarly, denote by $\partial_{L, o}^{u c} G$ the set of uniformly conical points in $\partial_{\lambda} G$ based at $o$. By Proposition 2.4. there exists one-to-one correspondence between $\Lambda_{L, o}^{u c} G$ and $\partial_{L, o}^{u c} G$.

The following is a version of Proposition 2.20 for generalized tight paths.

Proposition 5.12. There exist $l_{0}, D>0$ such that for any $L>0$, there exists $M=M(L)>0$ with the following property.

Denote $\alpha_{1}=[o, \xi]$ and $\alpha_{2}=[o, \eta]$ for $\xi \neq \eta \in \mathcal{C}_{L} G$. Let $\gamma$ be a generalized $(1, l)$ tight path for some $l \geq l_{0}$ with $\gamma_{-} \in \alpha_{1}$ and $\gamma_{+} \in \alpha_{2}$. If $d\left(o, \gamma_{-}\right), d\left(o, \gamma_{+}\right) \gg 0$, then there exists $z \in \gamma$ such that $d\left(z, \alpha_{1} \cup \alpha_{2}\right) \leq D$ and $d\left(z, \alpha_{i}\right) \leq M$ for $i=1,2$. Moreover, $|d(o, z)-d(o,[\xi, \eta])| \leq M$.

Proof. Let $l_{0}, c^{\prime} \geq c, K>0$ given by Lemma 5.10 such that the $K$-truncation $\tilde{\gamma}$ of a generalized $(c, l)$-tight $\gamma$ for $l \geq l_{0}$ is a $c^{\prime}$-quasi-geodesic. By Proposition 2.20. there exists an $\left(\epsilon_{c^{\prime}}, R_{c^{\prime}}\right)$-transitional point $z$ in $\tilde{\gamma}$ such that the conclusion of this Proposition holds. If the point $z$ lies on $\gamma$, then we are done. So below, we assume that $z \notin \gamma$, and then have two cases to consider as follows.

Let $L_{1}=L\left(\epsilon_{c}, R_{c^{\prime}}\right)$ be given by Lemma 2.17, where $\epsilon_{c^{\prime}} \geq \epsilon_{c} \geq \epsilon_{1}$ and they all satisfy Convention 2.18, Assume that $l_{0} \geq 2 L_{1}$. 
Case 1. The point $z$ lies in some $\left(\epsilon_{c}, K\right)$-component $\beta$ of a $(c, l)$-tight path $\gamma_{i}$. Then $\max \left\{d\left(\beta_{-}, \gamma\right), d\left(\beta_{+}, \gamma\right)\right\} \leq L_{1}$. Indeed, if not, then $\min \left\{d\left(z, \beta_{-}\right), d\left(z, \beta_{+}\right)\right\} \geq$ $L_{1}$. By applying Lemma 2.17 for the geodesic $\beta$, we have $z \in \tilde{\gamma}$ is $\left(\epsilon_{1}, R_{c^{\prime}}\right)$-deep in $X_{j}$. This is a contradiction, as $z$ is an $\left(\epsilon_{c^{\prime}}, R_{c^{\prime}}\right)$-transitional point in $\tilde{\gamma}$.

Case 2. The point $z$ lies in some $\left[y_{j}, x_{j+1}\right]$ for some $j$, where $\left[y_{j}, x_{j+1}\right]$ is given in Definition 5.9 of a generalized tight path. By the same reasoning as above, we apply Lemma 2.17 for the geodesic $\left[(\beta)_{-},(\beta)_{+}\right]$. Then $\min \left\{d\left(z, y_{j}\right), d\left(z, x_{j+1}\right)\right\} \leq L_{1}$.

Thus, we proved that $z$ has a distance at most $2 L_{1}$ to a point in $\gamma$. The conclusion follows as a consequence of Proposition 2.20.

The main result of this subsection is the following.

Proposition 5.13 (Visual Floyd/shortcut metric). There exists $0<\lambda_{0}<1$ such that the following holds for any $L>0$ and $\lambda \in\left[\lambda_{0}, 1\right)$.

We have

$$
\rho_{\lambda, o}(\xi, \eta) \asymp_{L} \lambda^{n}, \forall \xi \neq \eta \in \partial_{L, o}^{u c} G
$$

and

$$
\bar{\rho}_{\lambda, o}(\xi, \eta) \asymp_{L} \lambda^{n}, \forall \xi \neq \eta \in \Lambda_{L, o}^{u c} G
$$

where $n=d(o,[\xi, \eta])$.

Proof. Let us consider the shortcut metric case only. The Floyd metric case is similar and even easier.

Let $\alpha_{1}, \alpha_{2}$ be two $\left(\epsilon_{1}, R_{1}, L\right)$-transitional geodesic rays originating at $o$ and terminating at $\xi, \eta$ respectively.

Let $l_{0}, D>0$ be given by Proposition 5.12 , and we choose $\left.\lambda_{0} \in\right] 0,1[$ verifying Proposition 5.11 for $l=l_{0}$. Then by Propositions 5.12 and 5.11 there exists $M=$ $M(L)>0$ such that the following holds:

(1) For each $k>0$, there exists a sequence of generalized $\left(1, l_{0}\right)$-tight paths $\gamma_{k}$ with $\left(\gamma_{k}\right)_{-} \in \alpha_{1},\left(\gamma_{k}\right)_{+} \in \alpha_{2}$ and such that $\left(\gamma_{k}\right)_{-} \rightarrow \xi,\left(\gamma_{k}\right)_{+} \rightarrow \eta$ and

$$
\left|\mathfrak{l}_{\lambda, o}\left(\gamma_{k}\right)-\bar{\rho}_{\lambda, o}(\xi, \eta)\right| \leq 1 / k \text {. }
$$

(2) There exists $z_{k} \in \gamma_{k}$ such that $d\left(z_{k}, \alpha_{1} \cup \alpha_{2}\right) \leq D$ and $d\left(z_{k}, \alpha_{i}\right) \leq M$ for $i=1,2$. Moreover, $\left|d\left(o, z_{k}\right)-d(o,[\xi, \eta])\right| \leq M$.

Denote $u_{k}:=\left(\gamma_{k}\right)_{-}$and $v_{k}:=\left(\gamma_{k}\right)_{+}$.

Upper bound. Choose $x_{k} \in \alpha_{1}$ and $y_{k} \in \alpha_{2}$ such that $\max \left\{d\left(z_{k}, x_{k}\right), d\left(z_{k}, y_{k}\right)\right\} \leq$ $M$. Then for every point $t \in\left[x_{k}, z_{k}\right] \cup\left[z_{k}, y_{k}\right]$ we have

$$
d(o, t) \geq d\left(o, z_{k}\right)-M \geq n-2 M .
$$

Hence

We also have

$$
\max \left\{\mathfrak{l}_{\lambda, o}\left(\left[x_{k}, z_{k}\right]\right), \mathfrak{l}_{\lambda, o}\left(\left[y_{k}, z_{k}\right]\right)\right\} \leq M \cdot \lambda^{n-2 M} .
$$

$$
\max \left\{\mathfrak{l}_{\lambda, o}\left(\left[x_{k}, \xi\right]_{\alpha_{1}}\right), \mathfrak{l}_{\lambda, o}\left(\left[y_{k}, \eta\right]_{\alpha_{2}}\right)\right\} \leq \frac{\lambda^{\min \left\{d\left(o, x_{k}\right), d\left(o, y_{k}\right)\right\}}}{1-\lambda} \leq \frac{\lambda^{n-2 M}}{1-\lambda} .
$$

It follows that

$$
\begin{aligned}
\bar{\rho}_{\lambda, o}(\xi, \eta) & \leq \mathfrak{l}_{\lambda, o}\left(\left[x_{k}, \xi\right]_{\alpha_{1}}\right)+\mathfrak{l}_{\lambda, o}\left(\left[y_{k}, \eta\right]_{\alpha_{2}}\right)+\mathfrak{l}_{\lambda, o}\left(\left[x_{k}, z_{k}\right]\right)+\mathfrak{l}_{\lambda, o}\left(\left[y_{k}, z_{k}\right]\right) \\
& \leq 2 \lambda^{n-2 M}\left(\frac{1}{1-\lambda}+M\right) .
\end{aligned}
$$

Let $C_{1}:=2 \lambda^{-2 M}\left(\frac{1}{1-\lambda}+M\right)$. Then $\bar{\rho}_{\lambda, o}(\xi, \eta) \leq C_{1} \lambda^{n}$. 
Lower bound. Since $d\left(z_{k}, \alpha_{1} \cup \alpha_{2}\right) \leq D$, there exists $w_{k} \in \alpha_{1} \cup \alpha_{2}$ such that $d\left(z_{k}, w_{k}\right) \leq D$. Assume that $w_{k} \in \alpha_{2}$ for concreteness.

By Lemma 2.8, any segment of $\alpha_{2}$ is a Floyd geodesic. Since $v_{k} \rightarrow \eta$ and $d\left(o, w_{k}\right) \leq n+D$, we can assume that $w_{k} \in\left[o, w_{k}\right]_{\alpha_{2}}$ for all $k \gg 0$. So

$$
\begin{aligned}
\rho_{\lambda, o}\left(v_{k}, w_{k}\right)=\mathfrak{l}_{\lambda, o}\left(\left[w_{k}, v_{k}\right]_{\alpha_{2}}\right) & =\frac{\lambda^{d\left(o, w_{k}\right)}}{1-\lambda}-\frac{\lambda^{d\left(o, v_{k}\right)}}{1-\lambda} \\
& \geq \frac{\lambda^{d\left(o, z_{k}\right)+D}}{1-\lambda}-\frac{\lambda^{d\left(o, v_{k}\right)}}{1-\lambda} .
\end{aligned}
$$

We have

$$
\rho_{\lambda, o}\left(v_{k}, w_{k}\right) \leq \mathfrak{l}_{\lambda, o}\left(\gamma_{k}\right)+\mathfrak{l}_{\lambda, o}\left(\left[w_{k}, z_{k}\right]\right) .
$$

Since $d\left(z_{k}, w_{k}\right) \leq D$ we have

$$
\mathfrak{l}_{\lambda, o}\left(\left[w_{k}, z_{k}\right]\right) \leq D \cdot \lambda^{d\left(o, z_{k}\right)-D}
$$

Thus

$$
\begin{aligned}
\mathfrak{l}_{\lambda, o}\left(\gamma_{k}\right) & \geq \rho_{\lambda, o}\left(v_{k}, w_{k}\right)-\mathfrak{l}_{\lambda, o}\left(\left[w_{k}, z_{k}\right]\right) \\
& \geq \mathfrak{l}_{\lambda, o}\left(\left[v_{k}, w_{k}\right]_{\alpha_{2}}\right)-\mathfrak{l}_{\lambda, o}\left(\left[w_{k}, z_{k}\right]\right) \\
& \geq\left(\frac{\lambda^{D}}{1-\lambda}-\frac{D}{\lambda^{D}}\right) \lambda^{d\left(o, z_{k}\right)}-\frac{\lambda^{d\left(o, v_{k}\right)}}{1-\lambda} \\
& \geq\left(\frac{\lambda^{D}}{1-\lambda}-\frac{D}{\lambda^{D}}\right) \cdot \lambda^{M} \cdot \lambda^{n}-\frac{\lambda^{d\left(o, v_{k}\right)}}{1-\lambda} .
\end{aligned}
$$

Since $D$ does not depend on $L$ by Lemma 5.12 , there exists $1>\lambda_{0}>0$ such that

$$
\frac{\lambda^{D}}{1-\lambda}-\frac{D}{\lambda^{D}} \geq \frac{\lambda_{0}^{D}}{1-\lambda_{0}}-\frac{D}{\lambda_{0}^{D}}>0
$$

for any $\lambda \in\left[\lambda_{0}, 1\right)$. Let $C_{2}:=\left(\frac{\lambda_{0}^{D}}{1-\lambda_{0}}-\frac{D}{\lambda_{0}^{D}}\right) \cdot \lambda^{M}>0$. Note that $d\left(o, v_{k}\right) \rightarrow \infty$ as $k \rightarrow \infty$. By (35) and (37), passing to the limit when $k \rightarrow \infty$, we obtain

$$
\bar{\rho}_{\lambda, o}(\xi, \eta) \geq C_{2} \lambda^{n}
$$

for any $\xi \neq \eta \in \Lambda_{L, o}^{u c} G$ and any $L>0$. The proof is then complete.

Remark. (1) The fact that the constant $D$ does not depend on $L$ is crucial for the choice of $\lambda_{0}$ in 38 .

(2) This lemma gives an asymptotic formula for two uniformly conical points with respect to Floyd metric and shortcut metric. This could be used to give an alternative proof of Lemma 3.14 but cannot be derived from (the proof of) Lemma 3.14 .

\section{Appendix: Visual metrics and Floyd metrics are Bilipschitz EQUIVALENT}

The aim of the Appendix is to give a short proof that the visual Gromov metric $\nu_{a, o}$ and the Floyd metric $\rho_{\lambda, o}$ on the boundary $\partial X$ of a $\delta$-hyperbolic graph $(X, d)$ are bilipschitz equivalent for some choice of parameters $a$ and $\lambda$. This fact, mentioned in the Introduction, is often considered as a folklore, however we have not found a complete proof of it in the literature (see e.g. [18, Lemma 7.2.1] and the key inequality after it, or [6. Formula (1.3)], in both cases the fact is stated without proof). 
Recall the definition of the Gromov visual metric $\nu$ on $\partial X$. For a real parameter $a>0$ set $\delta_{a, o}(\xi, \eta)=e^{-a(\xi \mid \eta)}$, where $(\xi \mid \eta)$ denotes the Gromov product for the basepoint $o$. Let

$$
\nu_{a, o}(\xi, \eta)=\inf \left\{\sum_{i=0}^{n} \delta_{a, o}\left(c_{i-1}, c_{i}\right): c_{i} \in \mathcal{C}_{\xi, \eta}\right\},
$$

where $\mathcal{C}_{\xi, \eta}$ is the set of chains of points in $\partial X$ such that $c_{0}=\xi$ and $c_{n}=\eta$. If $a<\frac{\ln 2}{6 \delta}$ then $\nu_{a, o}$ is a metric on $\partial X$ satisfying the following inequality [17, Proposition 7.10]:

$$
\left(3-2 e^{a \delta}\right) \cdot \delta_{a, o}(\xi, \eta) \leq \nu_{a, o}(\xi, \eta) \leq \delta_{a, o}(\xi, \eta)
$$

Remark. We note that an inequality similar to 40 where the metric $\nu$ is replaced by the Floyd metric (which is our goal now) is formally stated in [23, page 5] but no justification is given.

For the Gromov product the following inequality is true [7, Lemma 2.7]:

$$
e^{-4 a \delta} \cdot \delta_{a, o}(\xi, \eta) \leq e^{-a d(o,[\xi, \eta])} \leq \delta_{a, o}(\xi, \eta)
$$

where $d(o,[\xi, \eta])$ is the distance in $X$ from $o$ to the union of all geodesics between $\xi$ and $\eta$. The inequalities 40 and 41 imply:

$$
\nu_{a, o}(\xi, \eta) \asymp C_{1} e^{-a \cdot d(o,[\xi, \eta])}
$$

for the constant $C_{1}=\max \left\{e^{4 a \delta}, 3-2 e^{a \delta}\right\}$.

The following proposition provides the bilipschitz equivalence between the visual metric and the Floyd metric on the boundary of $X$.

Proposition 6.1. Let $(X, d)$ be a $\delta$-hyperbolic graph. There exist a constant $a_{0}$ such that for any $\left.a \in] 0, a_{0}\right]$ there exists a constant $C$ for which

$$
\forall \xi, \eta \in \partial X: \nu_{a, o}(\xi, \eta) \asymp_{C} \rho_{\lambda, o}(\xi, \eta),
$$

where $\lambda=e^{-a}$.

Proof. By 39 and 42 we have

$$
\nu_{a, o}(\xi, \eta)=\inf _{\mathcal{C}_{\xi, \eta}} \sum_{i=0}^{n}\left\{\lambda^{d\left(o,\left[c_{i-1}, c_{i}\right]\right)}: c_{i} \in \mathcal{C}_{\xi, \eta}\right\} \leq C_{1} \rho_{\lambda, o}(\xi, \eta) .
$$

Indeed $\rho_{\lambda, o}$ is obtained by taking infimum of the expression 39 over the subset of $\mathcal{C}_{\xi, \eta}$ given by the sets of vertices of paths between $\xi$ and $\eta$ (see Section 2.2).

To prove the opposite inequality, we need to use the $\delta$-thin triangle property. Consider a geodesic triangle with vertices $o, x, y$ in $X$. There exists a $\delta$-center $c$ on $[x, y]$ such that $d(c,[o, x]) \leq \delta$ and $d(c,[o, y]) \leq \delta$. For notational simplicity we ignore a small uniform difference between different hyperbolicity constants (see e.g. [17, Proposition 2.21]), and denote all of them by $\delta>0$. Since $|d(o, c)-d(o,[x, y])|$ is uniformly upper bounded to simplify the notations again we assume that $d(o, c)=$ $d(o,[x, y])$. 
Choose $x^{\prime} \in[o, x]$ and $y^{\prime} \in[o, y]$ so that $\max \left\{d\left(c, x^{\prime}\right), d\left(c, y^{\prime}\right)\right\} \leq \delta$. We have $\min \left\{d\left(o,\left[x^{\prime}, c\right]\right), d\left(o,\left[y^{\prime}, c\right]\right) \geq d(o,[x, y])-\delta\right.$. Hence the Floyd length $\mathfrak{l}_{\lambda, o}\left(\left[x^{\prime}, c\right]\right)$ of $\left[x^{\prime}, c\right]$ is at most

$$
\delta \cdot \lambda^{d\left(o,\left[x^{\prime}, c\right]\right)} \geq \delta \cdot \lambda^{d(o,[x, y])-\delta}
$$

and similarly for $\mathfrak{l}_{\lambda, o}\left(\left[y^{\prime}, c\right]\right)$. Since $\left[x^{\prime}, x\right]$ and $\left[y^{\prime}, y\right]$ are Floyd $\rho_{\lambda, o}$-geodesics (Lemma 2.8) we have

$$
\mathfrak{l}_{\lambda, o}\left(\left[x^{\prime}, x\right]\right)=\frac{\lambda^{d\left(o, x^{\prime}\right)}}{1-\lambda} \leq \frac{\lambda^{d(o,[x, y])-\delta}}{1-\lambda},
$$

and the same for $\mathfrak{l}_{\lambda, o}\left(\left[y^{\prime}, y\right]\right)$. Summing all up we obtain the following estimation for the Floyd length of $[x, y]$ :

$$
\begin{aligned}
\rho_{\lambda, o}(x, y) & \leq \mathfrak{l}_{\lambda, o}\left(\left[x^{\prime}, x\right]\right)+\mathfrak{l}_{\lambda, o}\left(\left[x^{\prime}, c\right]\right)+\mathfrak{l}_{\lambda, o}\left(\left[y^{\prime}, c\right]\right)+\mathfrak{l}_{\lambda, o}\left(\left[y^{\prime}, y\right]\right) \\
& \leq 2 \frac{\lambda^{d(o,[x, y])}}{1-\lambda}+2 \delta \cdot \lambda^{d(o,[x, y])-\delta} \\
& \leq C_{2} \cdot \lambda^{d(o,[x, y])}
\end{aligned}
$$

for the constant $C_{2}=\max \left\{\frac{2}{1-\lambda}, \frac{2 \delta}{\lambda^{\delta}}\right\}$. Passing to the limits when $x \rightarrow \xi \in \partial X$ and $y \rightarrow \eta \in \partial X$, and using (42) we obtain 43 for the constant $C=\max \left\{C_{1}, C_{2}\right\}$.

\section{REFERENCES}

1. C. Bishop and P. Jones, Hausdorff dimension and kleinian groups, Acta Math. 3 (1995), no. 179,1 ? 39 .

2. B. Bowditch, Convergence groups and configuration spaces, Geometric Group Theory Down Under (J. Cossey, C.F. Miller, W.D. Neumann, M. Shapiro, eds.), pp. 23-54, de Gruyter, 1999.

3. Relatively hyperbolic groups, Int. J. Algebra Comput. (2012), no. 22, p1250016.

4. M. Burger and S. Mozes, CAT(-1)-spaces, divergence groups and their commensurators, Journal of the American Mathematical Society (1996), no. 1, 57-93.

5. J. Cannon, The combinatorial structure of cocompact discrete hyperbolic groups, Geom. Ded. (1984), no. 2, 123-148.

6. M. Coornaert, Mesures de Patterson-Sullivan sure le bord d'un espace hyperbolique au sens de Gromov, Pac. J. Math. (1993), no. 2, 241-270.

7. M. Coornaert, T. Delzant, and A. Papadopoulos, Géométrie et théorie des groupes. les groupes hyperboliques de Gromov, Lecture Notes in Mathematics, Springer-Verlag, 1990.

8. F. Dal'bo, M. Peigné, J.C. Picaud, and A. Sambusetti, On the growth of quotients of Kleinian groups, Ergodic Theory and Dynamical Systems 31 (2011), no. 3, 835-851.

9. W. Floyd, Group completions and limit sets of Kleinian groups, Inventiones Math. 57 (1980), 205-218.

10. D. Gaboriau and F. Paulin, Sur les immeubles hyperboliques, Geom. Dedicata (2001), 153-197.

11. V. Gerasimov, Expansive convergence groups are relatively hyperbolic, Geom. Funct. Anal. 19 (2009), 137-169.

12. __ Floyd maps for relatively hyperbolic groups, Geom. Funct. Anal. (2012), no. 22, 1361 -1399 .

13. V. Gerasimov and L. Potyagailo, Quasi-isometries and Floyd boundaries of relatively hyperbolic groups, J. Eur. Math. Soc. 15 (2013), 2115 - 2137.

14. Non-finitely generated relatively hyperbolic groups and floyd quasiconvexity, Groups, Geometry and Dynamics 91 (2015), 95-135.

15. _ Quasiconvexity in the relatively hyperbolic groups, Journal für die reine und angewandte Mathematik (Crelle journal) 710 (2016), 95-135.

16. Similar relatively hyperbolic actions of a group, International Mathematics Research Notices (2016), no. 7, 2068-2103.

17. E. Ghys and P. de la Harpe, Sur les groupes hyperboliques d'après Mikhael Gromov, Progress in Math., Birkaüser, 1990. 
18. M. Gromov, Hyperbolic groups, Essays in group theory (S Gersten, editor), vol. 1, pp. 75-263, Springer New York, 1987.

19. A. Karlsson, Free subgroups of groups with non-trivial Floyd boundary, Comm. Algebra. 31 (2003), 5361-5376.

20. P. Mattila, Geometry of sets and measures in Euclidean spaces: Fractals and rectifiability, Cambridge Studies in Advanced Mathematics, 44, Cambridge University Press, Cambridge, 1995.

21. S. Patterson, The limit set of a Fuchsian group, Acta Mathematica (1976), no. 1, 241-273.

22. F. Paulin, On the critical exponent of a discrete group of hyperbolic isometries, Differential Geom. Appl. (1997), 231236.

23. M. Puls, The first $l^{p}$-cohomology of some groups with one end, arXiv:math/0509171, 2005.

24. B. Stratmann, The exponent of convergence of Kleinian groups; on a theorem of Bishop and Jones, Proceedings of the Conference 'Fractal Geometry and Stochastics' (Friedrichroda, Germany, 2003); Progress in Probability, vol. 57, p. 93?107, 2004.

25. D. Sullivan, The density at infinity of a discrete group of hyperbolic motions, Publ. Math. IHES (1979), 171-202.

26. P. Tukia, Conical limit points and uniform convergence groups, J. Reine. Angew. Math. 501 (1998), 71-98.

27. W. Yang, Patterson-Sullivan measures and growth of relatively hyperbolic groups, Preprint, 2013.

28. _ Growth tightness for groups with contracting elements, Math. Proc. Cambridge Philos. Soc 157 (2014), $297-319$.

29. _ Peripheral structures of relatively hyperbolic groups, Journal für reine und angewandte Mathematik 689 (2014), 101-135.

Leonid Potyagailo, UfR de Mathématiques, Université de Lille 1, 59655 Villeneuve D'ASCQ CEDEX, France

E-mail address: potyag@math.univ-lille1.fr

Beijing International Center for Mathematical Research (BICMR), Beijing UniverSity, No. 5 Yiheyuan Road, Haidian District, Beijing, China

E-mail address: yabziz@gmail.com 